\title{
Die Lichtgeschwindigkeit ein „,neuer Gott" ? Von Einstein zu Kafka noch immer via Ludwig Hopf
}

„Ein Körper kann sich nicht mit Lichtgeschwindigkeit bewegen“1: Der Physikprofessor Ludwig Hopf, der diesen zentralen Satz geschrieben hat, spielte seine Rolle, wo es um Kafkas Begegnung mit Albert Einsteins neuem Weltbild ging. Hierbei kann von „Spekulation“ die Rede nicht sein. Denn der klavierspielende Hopf war wie gesagt im Jahr 1911 Einsteins Privatssistent in Prag gewesen und zugleich ein treuer Gefährte von Brod und Kafka in deren sozialen Kreisen in der Hauptstadt Böhmens. Darüber hat man neuerdings geschrieben: „An accomplished composer and pianist, Brod had a delicate discrimination and refine taste in matters of music. He set texts by Heine, Schiller, Flaubert and Goethe to music. (He had studied musical composition with Albert Schreiber, a student of Antonin Dvorak, and was proud of a distant relative, Henri Brod, a famous French oboist). Stefan Zweig remembered how , his small, girlish hands strayed gently over the keys of a piano. ' One evening in 1912, when Albert Einstein was teaching in Prague, Brod and the physicist played a violin sonata together. Leon Botstein, the American conductor and president of Bard College, speculates that for Brod, ,music facilitated what seemed impossible in politics: the forging of communication between the Czech and the German' "2 Wie immer weder Einstein umstandslos ein „Deutscher“, noch ebenso umstandslos Brod ein „Tscheche" gewesen war, erscheint der Hinweis von Wichtigkeit in unserem weiteren Kontext. Vielleicht vermag er dabei zu helfen, das viel beschrieene

\footnotetext{
${ }^{1}$ Ludwig Hopf, Die Relativitätstheorie, a. a. O., S. 56.

${ }^{2}$ Benjamin Balint, Kafka's last trial, New York, London, 2018, S. 21.
}

(C) Der/die Autor(en) 2021

B. Neumann, Umrisse einer Dritten Kultur im interdisziplinären Zusammenspiel zwischen Literatur und Naturwissenschaft, ELECTRISCHER PROMETHEUS. Umrisse einer Dritten Kultur im interdisziplinären Zusammenspiel zwischen Literatur und Naturwissenschaft, https://doi.org/10.1007/978-3-662-63204-8_19 
„Kafkaeske“ zu erklären, zu begründen, es womöglich gar „be-greifbar“ zu machen? Ludwig Hopf existierte schließlich beweisbar, wie immer er in der Kafka-Forschung bislang ein Unbekannter geblieben ist. War beweisbar Gast in Prag. Ließ darüber hinaus die hier zitierten Sätze über das neue Weltbild und die Lichtgeschwindigkeit, samt deren wissenschaftsgeschichtlicher Bedeutung, 1931 in einem Einführungsbuch im wohlrenommierten Wissenschaftsverlag Springer publizieren. ${ }^{3}$ Albert Einstein selbst hielt in Prag zweimal einen Vortrag über seine „Spezielle Relativitätstheorie“, jeweils mit Brod und Kafka als Zuhörern. Dennoch: Der entscheidende Überbringer der damals neuesten Nachricht vom neuen Weltbild der neuesten Physik war - Ludwig Hopf. Ein assimilierter Jude aus Nürnberg, mit musikalischen Interessen und Fähigkeiten wie die Dioskuren Brod und Kafka (oder meinethalben auch Gustav Mahler, Kafka erwähnt ihn im Tagebuch). Hopf hoffte damals auf eine Anstellung, womöglich auf eine Professur an der „Deutschen Universität“ in Prag. Zu realisieren war für ihn dann aber nur eine Privatdozenten-Stellung in Aachen, aus der erst später, nach dem Weltkriegsende 1918, eine Professur wurde. Ludwig Hopf musste deshalb sehr aktiv zugange sein im gesellschaftlich einflussreichen Kreis um Brod und Kafka, gewiß auch im „Fanta-Salon“, in dem auch sein Meister verkehrte und, wie zitiert, Violine spielte. Ludwig Hopf wusste selbstverständlich, dass Brod derjenige mit dem dichtesten sozialen Netzwerk in womöglich ganz Prag war. Also „courtisierte“, „umgarnte“ er Max Brod samt dessen unzertrennlichen Freund Kafka intensiv. Man brachte Wochen miteinander zu. Sie werden wohl miteinander gewandert, geschwommen (nackt, wie es die damalige „Jugendbewegung" befahl?), sie werden zusammen ins Kabarett und ins Bordell gegangen sein, neben ihren bereits erwähnten regelmäßigen Zusammenkünften in Prags so einflussreichem Fanta-Salon. Der Kontakt kann, er muss nahezu intim gewesen sein - aber eben deshalb womöglich auch potenziell zu ,ungesunden Verhältnissen“ führend, wie erwähnt. Er währte im Fall Einsteins lebenslang: Die Tochter der Familie Fanta wurde später in den USA eine von Einsteins zahl-

\footnotetext{
${ }^{3}$ Hopfs Buch erschien wie gesagt damals im Springer Verlag. Damit ist natürlich nicht der Berliner Axel Springer Verlag gemeint. Sondern dieser Springer nature Verlag bildet heute mit dem Metzler Verlag ein passendes Ensemble für ein Buch nicht nur über Kafka als einen „electrischen Prometheus“, sondern auch über den Kafka-Kenner und -Deuter Elias Canetti, das, von Gernot Wimmer und mir verfaßt, im Jahr 2020 erschienen ist: Elias Canetti in seiner Zeit, Stuttgart, Forschungsreihe Metzler.
} 
reichen Geliebten, ihr gegenüber wird das Physikgenie seine seltsam-mysthische Verbundenheit mit der magischen Stadt an der Moldau äußern. Sie war ihm geradezu die Pythia eines nebelhaft-berauschenden Prag-Kultes.

Hinzu kam die besondere Situation der Dioskuren: Brod war um 1911 zugange, den Roman Tycho Brahes Weg zu Gott fertigzustellen (in dem entweder Einstein oder Kafka, oder auch beide, das Modell zur Figur des Kepler abgegeben haben sollen), und er diskutierte diesen Text mit Kafka, der übrigens solche Verewigung gegenüber Felice infrage zog, bzw. direkt ablehnte. Franz Kafka sowie Max Brod wurden damals von Hopf darüber ins Bild gesetzt, dass Einsteins Revolution des Newton'schen Weltbildes mit der verglichen werden konnte, nein, musste, für die Charles Darwin im 19. Jahrhundert gesorgt hatte. Noch einmal abschliessend zusammengefasst: In des Pragers „Ansturm gegen die Grenze“ mithilfe seines „stehenden Marschierens“ wäre dann Einsteins ,göttliche“ Lichtgeschwindigkeit zugegen. Als eine ehedem frühromantisch-,,mesmeristische“ Größe, in der sich nunmehr das allermodernste Absolute eingenistet hatte, gewiss kein traditioneller jüdischer oder christlicher Gott mehr, aber eben doch ein Absolutes. Das wiederum war gar nicht so weit entfernt von der „leeren Transzendenz" nach Analyse und Meinung des Lyriktheoretikers (und seinerseits Romanisten und Arendt-Bekannten) Hugo Friedrich? Von Novalis' bekannter, romantischer Philosophie-Definition als so permanentem wie unstillbarem Verlangen nach einem Ganz Anderen gar nicht zu reden, auch nicht von Schellings hier einschlägiger Naturphilosophie. Es schließt sich ein Kreis, thematisch wie zeitlich. Derart kann sie sich ereignet haben, die Epoche schaffende „Wandlung des mechanischen zum elektronischen Weltbild“" (Originalton Hopf), die bereits in Kafkas frühem, hoch intensivem Interesse gegenüber der Elektrizität, im Verschollenen von 1914, aufscheint. Innerhalb des „mechanischen“ Weltbildes habe das Gravitationsfeld die Köper an ihrem Platz gehalten, so referierte Einsteins „Privatassistent“ damals in Prag. Nun aber träte der Elektromagnetismus an dessen Stelle und löste die alte Ordnung auf. Soziologisch kann man das, wie immer verkürzt, so fassen: Wer als Bauer geboren war, blieb im Prinzip lebenslang ein Bauer. Die Loyalität gegenüber dem König, der wie die Sonne im Zentrum steht (Ludwig XIV. in Versailles die Sonne tanzend bei Amtsantritt) sorgte für den gesellschaftlichen Zusammenhalt. Sie bestimmte das soziale Schwerkraftfeld. Auf diese Weise herrschte eine feste Ordnung. Nicht nur der Kosmos, sondern auch die (nun bereits absolutistische) Gesellschaft wird als ein Geflecht von Beziehungen begriffen, in dem die soziale Gravitationskraft auf alle Körper einwirkt, und diese festlegt in ihrer vorausbestimmten Bahn um das Zentrum herum. Die Musik dieser Weltordnung war, wie noch Kepler, diese Vorbildfigur für Brods Sicht des Albert Einstein, gewusst hatte, - die griechisch 
hergebrachte, harmonische Tonalität, eine von himmlisch musizierenden Sterne hervorgebrachte Tonfolge als Unterpfand umfassender kosmischer Harmonie gewesen. Jeder bedeutende Körper, jedes sozial hochstehende Individuum ist seinerseits sozusagen von einem Gravitationsfeld (eben dem der absolutistischen Macht) umgeben. Die Sonne hat ihre Planeten. Diese haben ihre Monde. Der Bauer hat seinen Knecht, und der womöglich seinen Hund. Freilich, die Feldstärke der Gravitation nimmt rasch ab mit dem wachsenden Abstand zu dem Körper, auf den sie wirkt. Das wurde mathematisch von Newton beschrieben. Und, wie gesagt, eine solche Gravitationsmechanik war, nicht nur dem Philosophen Leibniz zufolge, im Absolutismus die einzig göttlich „prästabilisierte“. Sie allein entsprang dem göttlichen Willen. Doch bei Kafka sind die Abstände nicht mehr konstant, die Wege wachsen, während man sie begeht, die Gesichter der Individualpartikel ändern sich wie ihre gesellschaftlichen Bahnen. Es hat folglich den Anschein, dass in den Romanen des Pragers, vor allem im Schloss, eine „Raumzeit“ zur impliziten Größe wird, wie sie beim beständig unternommenen, aber stets vergeblichen, eben „kafkaesken“ Annäherungsversuch an die „Grenze“ entsteht. Zeit hängt nun (im strikten Gegensatz zum Weltbild noch bei Newton/Leibniz) mit dem Raum zusammen; und der Raum mit der Zeit. Die Grundkategorien verschmelzen. Das „Kafkaeske“ entsteht, so wie die Atonalität nach der Tonalität entstand. Hopf schrieb: „Die Sicherheit, mit der man vor Einstein gewisse Aussagen über Zeitdauer, Raumabmessung usw. festhielt, rührte weder von Erfahrung noch von zwingenden Erwägungen her, sondern nur von der Gewohnheit des täglichen Lebens". ${ }^{4}$ Eben. Das hatte der Prager vernommen und seinerseits realisiert als moderner Romancier. Gerade die „Gewohnheit des täglichen Lebens“ durchbricht Kafkas neuer Roman-Typ verstörend, Angst und Orientierungslosigkeit erzeugend. Ein Einbruch des „Kafkaesken“ in unsere eigentlich ja immer noch Newton'sche Welt, eine „,neutönerische“ Auflösung der obsolet gewordenen Harmonie kosmischer Himmelsmusik. „Mit der Relativitätstheorie haben wir uns also auf den grundsätzlichen Standpunkt gestellt, unsre elektromagnetischen Erfahrungen höher zu werten wie unsere mechanische... In den mechanischen Gesetzen tritt somit eine Größe auf, die der Elektrodynamik entstammt, nämlich die Lichtgeschwindigkeit.... Insofern bedeutet die spezielle Relativitätstheorie die Krönung und Vollendung des elektrodynamischen Weltbildes." ${ }^{5}$ So also verhielt sich das, und Hopf betonte es so nachdrücklich wie

\footnotetext{
${ }^{4}$ Ludwig Hopf, Die Relativitätstheorie, a. a. O., S. 50.

${ }^{5}$ Ludwig Hopf, Die Relativitätstheorie, a. a. O., S. 72.
} 
begreifbar! Albert Einstein hatte 1911 in Prag, genauer: Am 24. Mai 1911, im „Fanta-Salon“, vor Brod und Kafka und eigentlich der intellektuellen Elite des deutschsprechenden Prag, seine ,Spezielle Relativitätstheorie“ selbst dargelegt. ${ }^{6}$ Das hatte bekannte, anerkannte, hier nicht in allen Einzelheiten zu referierende Folgen für Brods Tycho Brahes Weg zu Gott; vor allem die Figur des Kepler soll der Einsteins (oder doch Kafkas?) gleichen. Freilich, Einstein will Kafka in Prag nicht bemerkt haben, wogegen eher spricht, dass er später dessen Schloss offenbar gelesen/überflogen (und für ,pervers“ befunden) hat.

Im Fall Kafkas liegen die Verhältnisse weniger klar zu Tage. Den Prager hat jedenfalls eine erneute Visite Einsteins in Prag (der Heimatlose fühlte sich auf einschlägige Weise von der ,magischen Stadt“ angezogen, was Teil seiner Beziehung zur Fanta-Tochter Hannah später in den USA gewesen ist ${ }^{7}$ dazu angeregt, sich in sein Tagebuch (unter dem 10.IV.1922) zu notieren, dass ihn als Kind die Sexualität so wenig und so ,unschuldig“ interessiert hätte, wie heute die - Relativitätstheorie. „Nur Kleinigkeiten (aber auch die erst nach genauer Belehrung) fielen mir auf, etwa, dass gerade die Frauen, die mir auf der Gasse am schönsten und schönangezogensten schienen, schlecht sein sollten."8 Dann wäre also, gemessen an der noch hausfrauenhaft ,,anständigen“ Weltsicht Newtons und Leibniz', die neue Weltsicht Einsteins ihrerseits zwar am schönsten ,,angezogen,“ aber leider verderbt gewesen, was einander womöglich bedingte? Man war also aneinander interessiert, aber eben doch auch abgestoßen von der „Perversität“ des anderen, seinem „Umdrehen der hergebrachten Verhältnisse“? Dazu würde allerdings passen, was dann aus Kafkas Berliner Zeit kolportiert wird, also jenen Jahren kurz vor Kafkas Tod, wo er in der gleichen preussischen Hauptstadt wie auch Einstein wohnte. Einstein war hier Groß-Ordinarius (verweigerte aber immer noch die deutsche Staatsbürgerschaft, was ihm dann anlässlich der Verleihung des Nobelpreises Probleme machen würde). Der Mann fühlte sich als Jude, Schweizer und Pazifist, und musste bereits damals den Bau einer Atombombe als Konsequenz der Atomspaltung befürchten, schliesslich war er mit Curie wie Hahn persönlich gut und fachlich eng bekannt. Professor Einstein besaß in der Berliner Haberlandstraße seine Privatwohnung. Dort soll Kafka ihn besucht haben, wofür als Bürgschaft angegeben wird die allerdings vorhandene,

\footnotetext{
${ }^{6}$ Eine Beschreibung des Salons, Einsteins Auftritt etc. findet sich bei Michael D. Gordin, Einstein in Bohemia, a. a. O., S. 93 ff.

${ }^{7}$ Näheres bei Michael Gordin, Einstein in Bohemia, a. a. O., S. $256 \mathrm{ff}$.

${ }^{8}$ Franz Kafka, Tagebuch, 10.IV.1922.
} 
für Kafkas kommendes Tuberkulose-Sterben hoch wichtige Bekanntschaft mit dem in Ungarn geborenen Arzt Dr. Robert Klopstock, Kafkas späterem Vertrauten und Sterbehelfer, der seinerseits auch ein guter Bekannter Einsteins war. ${ }^{9}$ Oder haben die beiden einander doch verfehlt, war Einstein ausgegangen, als Kafka eintraf? Man fühlt sich in diesem Zusammenhang an jene exemplarische Geschichte einer stets verfehlten Begegnung erinnert, die zwischen Thomas Mann und Georg Lukacs Ereignis wurde, wo der eine immer gerade abgereist war, wenn der andere atemlos eintraf. Sie gilt womöglich auch für den PragLiebhaber Einstein und den Prag-Bewohner Kafka, jedenfalls, wenn man sie mit dem Namen Rudolf Steiner verbindet. Steiner war häufig Gast in Prag, und dann auch in Kafkas Tagebuch, dabei stets auf ironische Distanz gehalten. Das oben berichtete verhinderte Zusammentreffen geschah dort ebenso zwischen Einstein und Steiner. Hugo Bergmann als Fantas Schwiegersohn und Kafkas Schul- wie Studienkamerad hatte versucht, eine Begegnung zwischen Albert Einstein und Rudolf Steiner zu vermitteln. Doch die Vorlesungen, die Steiner (mit großer Resonanz übrigens, was Einstein allerdings anzog) in Prag hielt, sie schlossen genau vor der Ankunft Einsteins (der zwar die Bekanntheit Steiners goutierte, aber von Steiner Anthroposophie nicht viel hielt). Der Physiker hatte zuvor einmal Steiners Einlassungen zur „Okkulten Physiologie“ beigewohnt - und dabei genug gehört. Ausgerechnet Franz Kafka traf dann den Anthroposophen im gleichen Hotel, für das auch der Physiker gebucht war, am Tag eben der Abreise Steiners. Das „deutsche Prag“ war eben eine Insel mit begrenztem Umfang. Und das Resultat solchen sozialen Eifers? „You can be together with someone and still be alone“", schreibt Michae D. Gordin dazu. ${ }^{10}$ Gewiss, man wünscht seine Helden zusammen zu bringen, insbesondere, wenn beide unsere heutige Sicht auf die gesamte Welt verändert haben, und in der gesamten Welt zu bekannten Namen avanciert sind. Doch nicht um jeden Preis. Denn man begreift dieselben nicht allzu schlicht als „,good guys“, zwischen denen „something important happens"; ${ }^{11}$ sondern doch schon als bedeutende Akteure, am Projekt der Moderne arbeitend, insonderheit dann faszinierend, wenn in ihnen die „Zwei Kulturen“ einander begegnen. Solche epochale Begegnung erst macht es möglich, Franz

\footnotetext{
${ }^{9}$ Hubert Goenner, Einstein in Berlin, 1914-1933, München 2004, S. 211. Zur Rolle Klopstocks als Sterbehelfer Kafkas insgesamt Rotraud Hackermüller, Das Leben, das mich stört. Eine Dokumentation zu Kafkas letzten Jahren 1917-1924, Wien/Berlin 1984. Ferner Nicholas Murray, Kafka und die Frauen, Düsseldorf 2007, S. 327.

${ }^{10}$ Michael D. Gordin, Einstein in Bohemia, a. a. O., S. 8.

${ }^{11}$ Michael D. Gordin, Einstein in Bohemia, a. a. O., S. 9.
} 
Kafkas Invention des „Kafkaesken“ einsichtig zu machen. Erst dadurch wird möglich: Das Objekt der theoretischen Begierde, sozusagen ,auf frischer Tat ertappt“, bastelnd in seiner Werkstatt, um seinen so völlig neuen Blick auf die Welt zu präsentieren. Als Resultat solcher Konstruktionsarbeit erscheinen die rätselhaften, eben „kafkaesken“ Bälle des Junggesellen Blumfeld im Schreiben des Franz Kafka. Ihr Erscheinen begründet ein Stück „Dritter Kultur“ - hier entspringt womöglich auch eine ziemlich neue literaturtheoretische Form des „Beweisens“, als eines durch Gedankenoperationen einsichtig Machens und daneben immer noch konkreten Belege-Sammelns in Form der Geertz'schen „Thick Description“. Zumal Prag wie auch Wien in diesen Jahren die weltweit bedeutendsten Stätten epistomologischer Reflexion gewesen sind. Dem folgend, gewinnt man für die bohemische, habsburgerisch geprägte Metropole Prag jenen fremd-vertrauten Blick, der in ihr einen der Benjamin'schen „Ursprünge“ von Kafkas Schreiben zu erkennen möglich macht?

\subsection{Einstein in Prag und Besonderheiten der Moldau-Metropole. Eine alternative Geschichte der "Schwellen"-Stadt als "Ursprung" des "Kafkaesken"}

„Der Mensch tritt zum Duell mit Leben und Welt an. Erster Gang: die Eltern. Dann schickt das Leben andere Fechter vor: Mitschüler, Lehrer, die Mitbürger, das Publikum, die unergründliche Frauenwelt gegen den Mann. Lauter Feinde - oder zumindest lauter Gegenspieler, aus denen diejenigen, die es gut meinen, schwer herauszufinden sind (und schon dieses Herausfinden ist eben in gewissem Sinn eine Kampfhandlung, eine dem Menschen auferlegte Aktivität, eine Aufgabe, eine Lebensprüfung).“ (Max Brod, Franz Kafka, Biografie).

Die Stadt Prag hatte seit Jahrhunderten zwischen dem Tschechischen und dem Deutschen gestanden, ihre slavische Namens-Ableitung bedeutet denn auch "Schwelle“, entweder hin zum Osten, oder hin zum Westen Europas. Zwischen den Přemisliden und den Habsburgern, zwischen ,protestantischem“ Hussitentum und Habsburgerischem Katholizismus als einer „abendländischen“ Staatsreligion gelegen, ergaben sich für die „magische Stadt“ daraus besondere Prägungen, die weder Madrid, Paris, Wien oder Konstantinopel aufzuweisen vermochten, vom Spätling Berlin ganz zu schweigen. Diese Stadt hat Kafka mehr geprägt als seine persönliche Sozialisation, mehr jedenfalls als die längst zur Deuterlegende geronnenen Erzählung von der Unterdrückung des „ewigen Sohnes“ durch einen furchtbaren Alten. Selbst den Zürcher Wahlbürger Einstein 
hat sie lebenslang in ihrem Bann gehalten. Die liebliche, in ihre Flußschleife eingeschmiegte, in ihren „deutschen“ Bezirken hoch mondäne Moldaumetropole, Wiens Zwillingschwester und architektonisch so viel eigenständiger als beispielsweise deren andere Schwester Budapest, erscheint vor uns als die allererste Quelle von Kafkas so ganz besonderem Schreiben. Das ist konkret zu fassen mit Blick auf Prag als die Begegnungsstätte nicht nur zwischen Ost und West, sondern, zu Beginn des 20. Jahrhunderts, also bereits im Zeichen des sterbenden Doppeladlers, auch der zwischen Altem und Neuem Weltbild. Den Focus noch zu verengen: Es geschah, als Einstein hier seine Theorien zur Lichtgeschwindigkeit entwickelte, und Brod und Kafka diese via Hopf rezipierten. Aufseiten Einsteins entspricht dem das bereits referierte Faktum, dass dieser eingefleischte Internationalist, der auf seinen Schweizer Pass selbst im kaiserlichen Berlin, rabiat gegen alle preußische Korrektheit verstoßend, bestand, sich nie, und selbst nicht an seinem deutschen Geburtsort oder später dann in Jerusalem als der Hauptstadt Israels, so „heimisch“ gefühlt hat wie ausgerechnet in der Moldau-Stadt. Das aber hatten die weltweit unvergleichlichen alchimistisch-astrologisch-astronomischphysikalischen Entdeckungen gemacht, erst durch Brahe, Kepler, Kopernikus, und dann wieder durch Mach und Einstein, die hier erfolgt waren. Sie waren es, als stadtmythisch, archaisch vereinnahmende, die keine andere Stadt in der Welt zu bieten hatte, die, neben dem gemeinsamen Segeln (und gewiss auch den gemeinsamen Nächten) der Stoff bereitgestellt hatten, aus dem Einsteins Verhältnis zur Fanta-Tochter Hannah Fantova bestand, - in einem zweckrational bestimmten Amerika, das aber diesem Internationalisten andererseits gar nicht so sehr als „Exil“ fühlbar gewesen sein kann. Es bekümmerte Albert Einstein nicht so sehr, wo er „zu Hause“ war. In Prag aber war er es dennoch gewesen, wie immer sein ständiger, ununterbrochener Aufenthalt nur drei Semester umfasst hatte. Aufseiten Kafkas entspricht dem, dass der Nachtschreiber eigentlich Prag nie verlassen hat, trotz unablässig geschmiedeter Pläne, dies doch einmal zu erreichen. Als er seine Stadt verließ, geschah dies eigentlich, um zu sterben. Doch da ist andererseits auch jenes bereits zitierte Tagebuch-Bild: Wonach dem Prager die Relativitätstheorie so fremd und bunt erschienen sei, wie jene geschmückten Liebedienerinnen, bei denen er so viel häufiger gewesen ist, als die (ostjüdische) Impotenz-Legende es erahnen lässt. ${ }^{12}$

\footnotetext{
${ }^{12}$ So zuletzt bei Saul Friedländer, Franz Kafka, München 2012. Vgl. dazu Bernd Neumann, Der andere Franz Kafka, a. a. O., S. 34 ff.
} 
Auf ihre Art waren also beide einzig in Prag beheimatet. Es macht also von daher Sinn, daß der bereits schwer kranke Kafka im fernen Berlin doch noch den Professor Einstein besucht haben soll, mit dem er einen Bekannten teilte und auch eine frühe Bekanntschaft eben noch aus der Moldaumetropole. War solche Erzählung nicht das Gleichnis, die Metapher für beider Verbindung? Jedenfalls, wie vorausschauend, steht es bereits in einem Text Franz Kafkas angemerkt, der mit Von den Gleichnissen überschrieben wurde: Seine, also Kafkas, Texte würden später einmal, als hätte ein „Gläubiger“ oder ein „Weiser“ sie verfasst, als vorwiegend „gleichnishafte“ und „dunkle“ gelesen werden. Womit sie, so Kafka weiter, ,in irgendein sagenhaftes Drüben (verwiesen würden), etwas was wir nicht kennen, ... und was uns also hier gar nicht helfen kann ... Aber das womit wir uns jeden Tag abmühn, sind andere Dinge.“13 In der Tat. Es wurden „Gleichnisse" dort geschaffen, wo Kafka selbst sich noch durchaus an den provinziellen Realitäten seiner böhmischen Hauptstadt orientiert hatte, doch eben auch an deren neuesten naturwissenschaftlich-physikalischen Erkenntnissen, etwa der für ihn so bestrickend „bunten“ Einstein'schen Relativitätstheorie. Was geschähe mithin, wenn einen plötzlich der Mut zu der Naivität ankäme, Kafka in dem Sinn „wörtlich“ zu nehmen, dass man den Prager in einen direkten Zusammenhang mit den Diskursen aus seiner „Jetztzeit“ stellte, dabei die Grenze zur anderen Hälfte der Welt transdisziplinär überschreitend. Würde dann die Maus ihre Laufrichtung ändern? Und der Katze tatsächlich entkommen, der sie ja bei Kafka immer wieder in die Fänge läuft, auch bedingt durch die weltverengende Wirkung der Angst? Also Hegels ruhige Macht des „Wirklichen“ anstelle der fahrigen, umtriebigen Gewalt des „kabbalistischen“ Gleichnis-Machens, wie immer letzteres ebenfalls zum Prager Kosmos gehörte, und zwar unverzichtbar? Immerhin hat Franz Kafka in seinem Text Von den Gleichnissen weiterhin ausgeführt: „Würdet Ihr den Gleichnissen folgen, dann wäret Ihr selbst Gleichnisse geworden und damit schon der täglichen Mühe frei. Ein anderer sagte: Ich wette daß auch das ein Gleichnis ist. Der erste sagte: Du hast gewonnen. Der zweite sagte: Aber nur im Gleichnis. Der erste sagte: Nein, in Wirklichkeit; im Gleichnis hast Du verloren." ${ }^{14}$ Mit anderen Worten: Nur wer die Gleichnisse und ihr autonomes Kunst-Reich einerseits zwar erst nimmt, sie aber dennoch stets von der „Wirklichkeit“ her betrachtet, erhält nicht „nur“ im Gleichnis,

\footnotetext{
${ }^{13}$ Franz Kafka, Gesammelte Werke, Taschenbuchausgabe in sieben Bänden, hrsg. von Max Brod, Frankfurt/Main April 1976, Bd. 5, S. 72 (vgl. dazu Anmerkung 38).

${ }^{14}$ Franz Kafka, in: Gesammelte Werke in zwölf Bänden ..., hrsg. von Hans-Gerd Koch, Bd. 8, S. 131 f. (vgl. dazu Anmerkung 38).
} 
sondern auch „,in der Wirklichkeit“ Recht. Nicht mehr kabbalistische Beliebigkeit, sondern hermeneutisch-neuhistoristische Entschlüsselung der literarischen Texte, so wie ja auch nicht mehr astronomische Mythenbildung, sondern erst die konkrete Beobachtung durch Fernrohr oder Elektronenmikroskop bzw. Röntgenbestrahlung die unter der Oberfläche liegende „Wahrheit“ ans Licht bringt.

Welche Konstellation im Kräfteparallelogramm aus zeitgenössischen sozialen Energien bestimmte damals die Prager Geburtsstätte des kommenden altösterreichischen Beamten Franz Kafka? Die ersten Schreie, die der älteste Sohn und Statthalter des aufstrebenden Handelsherrn Hermann Kafka von sich gab, ertönten an der Grenze zwischen der „Josefstadt“, also dem ehemaligen, nun fast völlig beseitigten Ghetto, und dem Altstädter Ring, dem Zentrum der mächtigen Handelsstadt und Kulturmetropole Prag, dieser ,goldenen“, beziehungsweise „hunderttürmigen“ Hauptstadt Böhmens. Böhmen seinerseits war ein eigenes, ganz besonderes Terrain. Der habsburgerische Statthalter, Graf von Thun, war sich seinerzeit ganz sicher: „Ich bin weder Deutscher, noch Tschech', ich bin Böhme“. ${ }^{15}$ Auch eine „altösterreichische“ Standortbestimmung? Denn diese Provinz und ihre schon bald berühmte Prager Universität hatten spätestens seit dem Beginn des 15. Jahrhunderts die Kainsmale eines erbitterten Sprachen- und Nationalitätenstreits an sich getragen. Hier erschien das Habsburger Imperium „altösterreichischer“ und mithin umkämpfter als selbst im Wien Grillparzers. Hier wurde alles kontrovers diskutiert, was Europas Entwicklung bestimmte, von der „Geburt“ eines „nationalen Sozialismus“ samt eliminatorischer antisemitischer Züge, bis hin zur Geburt eines neuen Weltbildes, entwickelt von einem Pazifisten; und das alles um das Jahr 1900 herum als des Geburtsjahres der allerletzten Moderne, die, wie gezeigt, noch der Heutige Botho Strauß für schlechthin unvergänglich hält. Hieraus entstand Kafkas Werk als der letzte große Beitrag Europas zur Weltliteratur, in der Verwaltungssprache der Habsburger Monarchie gehalten, die ihr Autor sich freilich wunderbar geschmeidig zu machen wusste. Während das geistige und künstlerische Antlitz Böhmens unter Kaiser Karl IV. übernational-abendländische Züge trug, entwickelte sich die neuhochdeutsche Schriftsprache durch die Prager Kanzlei. Es entfaltete sich das Tschechische zur gleichen Zeit zur gepflegtesten slawischen Schriftsprache - während die Provinz sich, soziologisch-politisch betrachtet, erst zu einem konstitutionellen Ständestaat entwickelte, um danach ganz rasch zum bedeutendsten Industriegebiet der Donaumonarchie zu mutieren - und zum blutigsten Kampfgebiet zweier immer

\footnotetext{
${ }^{15}$ Bei Michael D. Gordin, Einstein in Bohemia, a. a. O., S. 5.
} 
noch „habsburgerisch“ verklammerter Nationen zugleich. Also: Die Geburt des Sohnes Franz Kafka gerade an dieser Stätte glich in gewissem Sinne einer Landnahme, gedacht in der Tradition der (jüdisch-römischen!) Flavius-JosephusLegende, wie sie von Elias Canetti in Masse und Macht entfaltet wurde. Der Fremde, der das Eigene erst unverwechselbar sichtbar zu machen vermochte, als er kein Fremder mehr war. Ein ausländischer „Landvermesser“, ganz wie im späteren Schloss-Roman, gerade vom allzu platten Lande in die hoch getürmte Stadt gekommen, und hatte da bereits eine ganz neue Landmarke markiert? Kafkas Geburtshaus stand jedenfalls mächtig inmitten der Metropole. Setzte seine architektonischen Herrschafts-Zeichen, „mit der Rückseite an die von K. I. Dientzenhofer erbaute Nikolauskirche gelehnt, das Geburtshaus Franz Kafkas, ,Zum Turm' (ù veže', Konskriptionsnummer I/27). Es war in früheren Zeiten Besitz des mächtigen Klosters Strahov, zu dessen Dependancen die St. Nikolaus-Prälatur gehörte, der 1629 das Haus ,Zum Turm ' einverleibt wurde.“ Dieses prächtige und traditionsstolze Haus, dessen Bild soeben wiederbelebt wurde durch Zitieren einer ihrerseits für die Kafka-Biographik bahnbrechenden Quelle, ${ }^{16}$ in dem damals der neueste Stadtschreiber der ,goldenen Stadt“, ihr Chronikverfasser fürs Neunzehnte und vor allem Zwanzigste Jahrhundert, ein Flavius Josephus redivivus: Franz Kafka, zur Welt kam - dieses Haus hatte zuvor dem Prager Stadtschreiber Ulrich von Falkenau (und später dann dem obersten Stadtschreiber des Altstädter Bezirks, dem Freund des späteren Papstes Aeneas Silvius, nämlich Johann Toušek) gehört. Erst einem deutschen, und dann einem tschechischen Notabeln. Aber beide mit der Herstellung der Schrift befasst. Wegmarken des gesamteuropäischen Schicksals zu Beginn des 20. Jahrhunderts, seit dem Jahre 1910 durch den Halley'schen Kriegskometen markiert, mit dem dann auch Kafkas Tagebuchschreiben beginnen würde, Apokalyptisches verheißend. In solchem gegensätzlichen Miteinander schlug sich eine andere Geschichte Prags nieder, die viel näher an jener mythischen erschien, die schliesslich Albert Einstein nach Prag zog. Sie gilt es nun zu rekonstruieren (nachdem die andere, kulturgeschichtlich ausgerichtete Geschichte Prags, in anderen Zusammenhängen bereits geschildert wurden). ${ }^{17}$

Wie war Einstein nach Prag gekommen? Wie konnte es sein, dass dieser Mann, ein rational denkender Naturwissenschaftler, sich später dann auf nahezu

\footnotetext{
${ }^{16}$ Klaus Wagenbach, Franz, Kafka, Bern 1958, S. 13.

${ }^{17}$ In: Ebenfalls Klaus Wagenbach, Franz Kafka, a. a. O., daneben Hartmut Binders Arbeiten inklusive Christoph Stölzs Beiträgen, und schliesslich Bernd Neumann, Der andere Franz. Kafka, Würzburg 2018, S. 71 ff.
} 
mystische Weise dieser Stadt verbunden fühlte, und das nach lediglich zweisemestrigem Aufenthalt? Diese Fragen zu beantworten, beginnen wir mit der Berufungsgeschichte, wie sie uns als die von Einstein in Bohemia überliefert ist. Seit alters her besteht ein Konsens darüber, dass das Leben in Prag, verglichen mit dem im Rest der Welt, ein ganz besonderes sein soll. Der Prager Philosoph Carl Stumpf hat diesen Konsens, in Zusammenhang mit Einsteins Übersiedelung nach Prag übrigens, wie folgt ausgedrückt: „Das Leben in Prag hatte mich physisch wie psychisch so beansprucht, dass ich die Rückkehr zu ordentlichen und ruhigen Verhältnissen als eine Art von Erlösung begrüßte. Die Leute in Wien und auch die bei uns in Deutschland hatten damals keine Ahnung davon, wie sich Deutsche in Prag fühlten."18 Einstein, nach Prag berufen, erfuhr diesen Ausnahmezustand, und der sollte ihn dann lebenslang an den Ort des offenbar mystisch Besonderen fesseln, wobei die Intensität dieser Fesselung mit anwachsendem Alter immer nur noch zunahm! Die Geschichte dieser Bezauberung gilt es nachzuerzählen, als Erzählung von einem nicht restlos erklärbaren Verfallen-Sein. Sie begann schlicht mit einem Ruf auf eine akademische Position, spektakulär durch die Person des Berufenen und den Ort der Berufung, gewiss, aber doch eigentlich nicht ungewöhnlich. Albert Einstein hatte damals bereits eine ganz ungewöhnliche Karriere als Physiker hinter sich, der Mann war andererseits doch bereits 32 Jahre alt, als er sich im Jahr 1910 dazu entschloss, sich nach Prag zu bewerben. Die Gründe dafür sind nicht restlos rekonstruierbar. Seine rumänische Frau samt Familie (spätere feministische Versuche, der Physikerin die „eigentliche“ Entdeckung der Relativitätstheorie zu übertragen, sind gescheitert) waren damals, im schönen Zürich, entschieden dagegen. Auch Einstein selbst war gar nicht unglücklich am Zürichsee, doch hatte er wohl Prag samt seiner Karriere im Kopf als traditionell den zentralen Ort naturwissenschaftlicher Forschung. Dessen Aura erlag der Mann, einmal für immer. Hinzu kamen Einsteins enge Kontakte mit Physikern vor Ort, etwa Ernst Mach, und vor allem mit dem Übervater aller modernsten Physik, Max Planck. Der saß zwar in Berlin, bestärkte ihn aber entscheidend in seinem Entschluss, nach Prag zu gehen. Letzterer würde dann auch eine zentrale Rolle bei seiner Berufung spielen (nachdem so gut wie alle entscheidenden Forschungsresultate Einsteins, auf dessen Weg hin zur „Speziellen Relativitätstheorie“, in Hahns zentralen Annalen der Physik veröffentlich worden waren). Und nicht nur

\footnotetext{
${ }^{18}$ Michael D. Gordin, Einstein in Bohemia, a. a. O., S. 20. (Meine Übersetzung des dort in Englisch wiedergegebenen Zitats).
} 
dies: Einstein war ein Stück weit auch ein Spieler; die Erforschung des Neuen war sein eigenstes Element, wissenschaftlich wie lebensgeschichtlich. Für Prag sprachen also der Ruf der Stadt neben dem erhöhten Salär. Ferner war seine Ehebeziehung mit Mileva bereits so strapaziert, dass der Widerstand der Ehefrau eher stimulierend gewirkt haben kann (etwa so, wie deren Widerwillen, Einsteins Geigenspiel in beider durch Kacheln hellhöriger Zürcher Küche zu lauschen, ersterem bei seinem Spiel immer nur anstachelten; die Haare dabei wie ein Zigeuner aufgestellt, „electrisch“ eben). Das Genie wollte damals nach Prag, blieb aber dann nur drei Semester, - um erst hinterher zu merken, ,how total his immersion was. "19 Ihm widerfuhr ein Verfallen-Sein an diese „magische Stadt“, das seinerseits zentraler Topos Prager Literatur immer schon gewesen war (und ist, siehe in unseren Tagen Milan Kundera, dessen Werk samt Romantheorie nicht denkbar erscheint ohne den Einfluss seiner Geburtsstadt samt seines auf Prager Weise musikkundigen Vaters). Angefangen mit Gustav von Meyrincks so bekannt gewordenem Golem, bis hin zu Libuśe Monikovás Die Fassade besitzt dieser Satz seine Wahrheit. Es darf aber auch die besondere Geschichte und Ausstrahlung der Universität, an die der Mann sich 1910 bewarb, nicht unterschätzt werden. Diese älteste Universität in Mitteleuropa, Gründungsjahr 1348, durchzogen von wissenschaftlichen, religiösen, dann nationalen Spannungen bis hin zu universitären Kriegszuständen, war zu Einsteins Zeiten immer noch ein weltbekannter Mythos. War zudem weltweites Zentrum für epistomologische Fragen vor allem in Sachen einer Zusammenarbeit zwischen den beiden Wissenschaftskulturen, freilich gerade hierin, seit etwa der Mitte des 19. Jahrhunderts, eigentlich nur noch durch die Linse des kalten Bürgerkriegs der Tschechen gegen die „Deutschen“ und vice versa zu betrachten. Ein Tatbestand, ohne den auch Kafkas gesamtes Schreiben gar nicht zu verstehen ist, ${ }^{20}$ und Einstein würde, wie immer wider seinen Willen, in ihn hineingezogen werden. „All of Einstein's interactions with Prague, from before he arrives until his death, were in some way mediated by this university ... One cannot understand why the faculty offered a position to Einstein, what that encountered when he accepted, nor what transpired afterward without fairly deep background about this institution." ${ }^{21}$ Den Aspekt abzurunden: Diese alte, seit je der Suche nach einer „Weltformel“ verpflichtete Universität faszinierte

\footnotetext{
${ }^{19}$ Michael D. Gordin, Einstein in Bohemia, a. a. O., S. 20.

${ }^{20}$ Auch dazu Christoph Stölzl, Kafkas böses Böhmen, München 1975. Hierüber besteht eigentlich Einigkeit in der gesamten Kafka-Forschung, der englischen wohl noch entschiedener als in der (bundes) deutschen.

${ }^{21}$ Michael D. Gordin, Einstein in Bohemia, a. a O., S. 21.
} 
folglich auch den Zürcher Einstein, als er sich 1910 an der dortigen „Deutschen Universität“, Philosophische Fakultät, bewarb. Der Wissenschaftler war bereits in der Ausarbeitung der „Allgemeinen Relativitätstheorie“ begriffen. Die Magie des Ortes würde ihn bis ans Lebensende verfolgen, der Ruhm seiner dort erarbeiteten Relativitätstheorie gleichermassen.

Das dargestellte „elektrisierende“ Verhältnis zwischen Bewerber und Universität (mit dem Pager Autor Kafka als bis heute verborgenen Dritten im Bunde), besaß seine Entsprechung im avancierten Gegenstand der Berufungsverhandlungen. „Modern theoretical physics has experienced its most powerful stimulus from the study of electricity.“22 Es ging dabei implizit um die Installation eines komplett neuen Weltbildes, was auch Max Planck seinerseits realisierte und als selbstständigen philosophischen Sachverhalt begriff. Der Berliner Nestor der damals noch weltbedeutenden deutschen Physik hatte ja alle Arbeiten Einsteins aus dessen „miracle year“ 1905 in seinen Annalen der Physik publiziert, war dann ab 1906 auch offizieller Herausgeber geworden; und er wusste durchaus, wen er da empfahl. Schrieb also nach Prag über die Bedeutung von Einsteins Weltbild-Revolution die Sätze: "With the extend and depth of the revolution in the domain of the physical worldview summoned by this principle one can only compare that caused by the introduction of the Copernican world system. “23 Ein so atemloser wie umstürzender Satz. Der dennoch nicht umstandslos zur Besetzung der Professur durch Albert Einstein führte. Nun kam nämlich erst einmal Wien ins Bild, und damit „Kakanien“. Auf Platz zwei der Liste der Philosophischen Fakultät stand Gustav Jaumann, ein Mach-Schüler besonderer Prägung, damals ordentlicher Professor der Physik in Brünn; jemand, der (unglaublich, aber wahr, und dann auch noch Jahre nach Einsteins Durchbruch zur Weltgeltung) leugnete - dass es Atome überhaupt gäbe! „When Jaumann died in 1924, he was in the midst of composing a 2,000-page textbook filled with continuous differential equations to demonstrate a physics with united forces and without any atomic particles - the position of a borderline heretic.“24 Dieser wohl tatsächlich psychopathische Häretiker der modernen Physik, der aber, wir werden es noch sehen, über seinen besonderen Stil verfügte, über die bestrickende Grandezza des vollständig Gestrigen, wurde vom

\footnotetext{
${ }^{22} \mathrm{Im}$ Ausschreibungstext des Berufungskomitees; bei Michael D. Gordin, Einstein in Bohemia, a. a. O., S. 23.

${ }^{23}$ Michael D. Gordin, Einstein in Bohemia, a. a. O., S. 24, dort zitiert nach dem offiziellen tschechischen Bericht über die Berufung Einsteins nach Prag.

${ }^{24}$ Michael D. Gordin, Einstein in Bohemia, a. a. O., S. 40.
} 
zuständigen Wissenschafts-Minister in Wien, Karl Graf von Stürgkh (der Mann starb übrigens nicht viel später, bei einem glanzvollen Festessen, durch die Kugel eines „revolutionären“ jüdischen Attentäters) an Einstein vorbei auf Platz Eins promoviert. Hinter dieser ministriellen Revolution standen jene, bereits damals beträchtlichen antisemitischen Kräfte, die, wie etwa Johannes Stark in Aachen, eine ,arische Physik“ anstrebten; mithin eine gegen die ,jüdisch spekulative“ der von Einstein, Hopf und Konsorten betriebenen. Es stand Einsteins Berufung nun plötzlich infrage - bis der Minister und Jaumann sich dann ihrerseits über die Frage der Besoldung entzweiten. Und Gustav Jaumann (als Mach-Schüler jemand, der wirklich wusste, welche Verhältnisse in Prag herrschten) seinerseits Rückgrat auf die besondere Weise bewies, dass er dem Minister zu verstehen gab, er könne keinesfalls Professor werden an einer Universität, deren Philosophische Fakultät - einen Atom-Gläubigen wie Einstein berufen wolle! Ende der „kakanischen“ Berufungs-Scharade; und: Dienstantritt Albert Einsteins am 3. April des Jahres 1911. Der nun doch noch Berufene brach, nach ausgiebigen Besuchen bei befreundeten Physikern unter anderem in Deutschland, nach Prag auf. Die Fakultät hatte ihren jungrevolutionären Star bekommen, und in ihm einen neuen Gefährten des nachtschreibenden Prager Beamten Franz Kafka, auch wenn der Neue sich kaum als „deutsch assimilierter“ Jude verstand. (In diesem Zusammenhang ist noch nachzutragen, dass Einstein, als er seiner Mutter mitteilte, der Minister wolle ihn nicht haben, seinen ,semitic descent“ dafür verantwortlich machte). ${ }^{25}$

Auf diese Weise war also Albert Einstein unter die Prager Universitätsleute gefallen, die er nur als deutschsprachige wahrgenommen haben kann, bei den nicht vorhandenen Kenntnissen des Tschechischen aufseiten des neu berufenen Genies. Der Neue samt seiner Familie erreichten Prag am 3. April 1911. Nach einigen Tagen im Hotel bezog man ein eigenes Apartment in einem (noch heute erhaltenen, damals neuen) Haus unweit der Moldau - auf deren Wellen auch Kafka zu blicken pflegte, wenn er morgens mit der Niederschrift (etwa des Urteils) fertig geworden war, bei aller Müdigkeit stolz sich streckend, dem eintretenden Dienstmädchen entgegensehend, als erwarte er Lob für sein asoziales Nachtschreiben. Albert Einstein hatte damals seinen Posten an der „Deutschen Universität" vor allem als ein Forschender bezogen. Der Physiker wird nahezu

\footnotetext{
${ }^{25}$ Carl Seelig, Albert Einstein: Leben und Werk eines Genies unserer Zeit, Zürich 1960, S. 117.
} 
seine gesamte Zeit zubringen mit Experimenten zur Lichtgeschwindigkeit, ihn durchaus zufriedenstellenden, was in unserem Zusammenhang verzeichnenswert erscheint. „The Speed of Light“ ist folgerichtig eine passende Überschrift für Einsteins Tätigkeit in Prag. ${ }^{26}$ Wir sind über Einsteins damaligen Gemütszustand, wie er wesentlich vom Erfolg seines Arbeitens abhing, durch zahlreiche Mitteilungen des Chefs an seinen Assistenten und Duo-Partner Hopf informiert, die Nähe beider zueinander zeigte sich auch darin. Albert Einstein, über die Natur des Lichts arbeitend, befand sich nun ständig zwischen dem Mythos der Moldaumetropole und seinen täglichen, mühsamen, von Lehrverpflichtungen vor teilweise uninspirierten oder gar stupiden Studenten unterbrochenen, im Alltag unspektakulär sich hinschleppenden universitären Verpflichtungen. Sein Arbeiten wiederum geschah entweder in experimenteller oder in mathematischer Form; war stets aber konzentriert auf die Natur des Lichtes und verbunden mit der Frage nach der Erreichbarkeit der Lichtgeschwindigkeit. Die dargestellte enge Vertrautheit mit Hopf kann dabei bewirkt haben, dass sich dieser Sachverhalt auch an die Dioskuren Brod und Kafka vermittelte, wenn Hopf die beiden über die Theoriebildung seines Meisters informierte. Beispiele dafür finden sich: Die spektakulär annoncierte Tagung der Brüsseler „Solvay Konferenz“, Marie Curie war anwesend neben nahezu der gesamten Elite der damaligen Atomforschung, und der Womanizer Einstein nahm sie selbstverständlich auch als anziehende Frau wahr, diese Konferenz erlebte der künftige Atombomben-Gegner doch tatsächlich als den Brüsseler „Hexen-Sabbath.“27 Das teilte er auch Ludwig Hopf brieflich mit, und der vertraute Ton unterstreicht einmal mehr die Interessiertheit des Assistenten am Forschungsfortschritt des Genies: „Herausgekommen ist nichts in Brüssel, aber es war ein durchaus vergnügliches Spektakel.“28 Die skeptische Beurteilung lag darin begründet, dass Einstein in diesen Monaten an ganz anderem als an der Arbeit mit dem Uranatom interessiert war, um die es in Brüssel ja vor allem gegangen war. Er arbeitete an der Hypothese, wonach die hergebrachte orthodoxe Interpretation des Lichts als ausschließlich ein Wellenphänomen zu ergänzen sei. Das wiederum erweist sich als der hot spot seiner damaligen Forschung. Für den Beweis, dass Licht eben auch aus Partikeln bestand, würde der Mann 1921 den Nobelpreis erhalten. Solcher Erforschung des

\footnotetext{
${ }^{26}$ Das bei Michael D. Gordin, Einstein in Bohemia, a. a. O., S. 40.

${ }^{27}$ So an seinen Schweizer Freund Michele Besso in einem Brief vom 13.Oktober 1911 (aufbewahrt in: The Collected Papers of Albert Einstein, Princeton University).

${ }^{28}$ The Collected Papers of Albert Einstein, a. a. O. Geschrieben nach dem 20. Februar 1912, da befand sich Hopf bereits in Aachen.
} 
Lichts war Albert Einstein sozusagen auch freudianisch verpflichtet (der Vater als Lichtbringer einer deutschen Kleinstadt!), und eben die Fortschritte auf eben diesem Gebiet teilte er damals Ludwig Hopf eifrig mit. Der Nürnberger muss ihm also alles andere gewesen sein, als ein Faktotum nach Art des Wagner in Goethes Faust. Eben darin, in der Neubestimmung der Natur des Lichts, lag damals die tägliche Beschäftigung dieses außergewöhnlichen Kopfes, der, darin weniger eine Ausnahme, in der verkehrsreichen Weinberggasse in Prags Innenstadt seinen Sitz hatte, ein Gehalt von 8672 Kronen bezog, bei allerdings geringer Lehrbelastung und limitierter Laborarbeit - ein Beamter, der dabei war, ein Weltbild zu zertrümmern, dabei wie Kafka schreibend auf die Moldauwellen schauend. Oder auch: Wie einst Goethe in Rom, so war Albert Einstein er nun in dem, „was seines Vaters“ war, und er teilte das daraus entspringende Glücksgefühl mit seinem nahezu gleichaltrigen Assistenten, auch, als der gar nicht mehr in Prag weilte. Dies in Anschlag zu bringen ist wichtig, gerade auch mit Blick auf den damaligen Umgang zwischen Hopf und Brod (samt Dioskur Kafka); es ereignete sich sozusagen mitten im historischen Herzbereich des ohnehin mythischen Prag, dass Einstein nun seine neue kopernikanische Revolution in Angriff nahm. Während Brod damals bereits an seinem astronomischen Tycho-Roman schrieb, würde Kafka erst nach einigen Jahren den Blumfeld in Angriff nehmen. Albert Einstein wiederum sandte seine Forschungsresultate, die Natur des Lichts und die des statischen Gravitationsfeldes betreffend, im kommenden Jahr 1912 an Max Planck, zwecks Publikation in den dort erscheinenden Annalen, wie gewohnt. Der Maestro vermochte gegenüber Hopf zu jubeln: „Rigoros habe ich nunmehr die Theorie der Gravitation für das statische Feld entwickelt. Das Resultat ist wunderschön und erstaunlich einfach. Die Theorie von Abraham ist grundlegend falsch. Ich werde sicherlich ein Feder-Duell mit ihm haben." ${ }^{29}$ Das waren hohe Töne; hier feierte einer, was er als seinen Durchbruch zu bereits der „Allgemeinen Relativitätstheorie“ erlebte - und was ein wenig vorschnell war, wie wir heute wissen.

\footnotetext{
${ }^{29}$ The Collected Papers of Albert Einstein, a. a. O., ebenfalls nach dem 20. Februar an Hopf in Aachen.
} 


\subsection{Die Stadt als Gedächtnisraum. Sigmund Freud (und Aleida Assmann) als deren Theoretiker. Meyrinks Prag-Roman Der Golem als ein "surrealistisches", weil mit „Rassen“"energie elektrisch geladenes Erzählstück. Albert Einstein spielt auf seiner Geige Mozart}

Kein Zweifel, dass die spezielle Signatur einer solch besonderen Geburtsstadt das Schreiben des in ihr Geborenen beeinflussen, ja prägen kann. Das galt gewiss für Kafka; galt aber eben auch für Einstein, den Zugereisten, der an magischem Ort geradezu magische Fortschritte in seiner Theoriebildung erlebt haben wollte. Jener Albert Einstein, der (und dann bereits nach epochalen Erfolgen, etwa nach der Verleihung des Nobelpreises) stets wie magisch angezogen nach Prag zurückkehren wird als die Stadt, die er immer wieder besuchte. Also: Prag als besondere Stadt in ihren archäologischen Schichtungen, mithin als eine „elektrisierende“ Strahlenquelle, gespeist aus historisch erworbener Energie; eben der, seit der Antike bekannte, zuerst auf Athen wie Rom gemünzte „genius loci“. Solchen „genius loci“ zu spüren und zu würdigen, ist selbstverständlich nicht zu verwechseln mit schierer Behaglichkeit urbanen Lebens. Kafka litt erheblich unter den politisch-ethnischen Kämpfen seines Prag. Einstein dagegen befand die zeitgenössische Stadt Prag als den Ort eines, mit Zürich verglichen, eher zurückgeblieben, nahezu stupiden städtischen Lebens, das, auch durch den permanenten Vergleich mit Zürichs urbaner Lebendigkeit (und mit dem dort gegebenen Fehlen eines offenen Antisemitismus), er nicht so recht zu schätzen wusste. ${ }^{30}$ Hinzu kam die häusliche Situation mit einer ihm entfremdeten, dabei selbst "slavischen“ Frau, einer Serbin, die weder von den Prager Slaven, noch auch von den „deutschen“ Machthabern respektiert wurde. ${ }^{31}$ Die in ihrem wissenschaftlichen Ehrgeiz frustriert war, sich in Erinnerung an das so viel behaglichere Zürich verzehrte, und die sich zudem mit ihres Mannes außerehelichen Eskapaden permanent konfrontiert sah. Hinzu gesellten sich Ärgernisse des täglichen Lebens wie etwa dieses, dass die Moldau-Stadt in puncto Elektrifizierung zwar Zürich voraus war, aber nicht einmal genießbares Trinkwasser anzubieten hatte. Alles

\footnotetext{
${ }^{30}$ Interessant ist, dass der international so erfahrene Elias Canetti eine ganz ähnliche Erfahrung gemacht hat, die besondere, avancierte Bekämpfung des Antisemitismus in Zürich betreffend. Siehe dazu Bernd Neumann, Gernot Wimmer, Canetti in seiner Zeit, Stuttgart 2020, S. $52 \mathrm{ff}$.

${ }^{31}$ Dazu ebenfalls Michael D. Gordin, Einstein in Bohemia, a. a. O., S. 99 ff.
} 
musste abgekocht werden, was die häusliche Atmosphäre nicht verbesserte. Einstein sah zudem bei den Prag-Bewohnern immer wieder antisemitische Neigungen durchbrechen, tadelte deren Unterwürfigkeit, diagnostizierte bei manchen seiner Studenten interessenlose Trägheit. Und doch lehnte der Mann damals Rufe (wie den nach Utrecht) ab, unterzog sich in Prag pflichtgetreu seiner wissenschaftlichen Arbeit, und verkehrte daneben gesellschaftlich sehr rege mit Freunden, Bekannten, attraktiven Frauen, allen ehelichen Schwierigkeiten zum Trotz. Sein passioniertes Geigenspiel brachte ihn mit sehr vielen Bekannten, nicht nur aus dem Fanta-Kreis, zusammen. Immer schon ein gesellschaftsfreudiger Mensch, hielt es auch an der Moldau ganz so, wie zuvor am Zürichsee und bereits in Bern. Allerdings verkehrte er in der ,goldenen Stadt“" ausschließlich mit den dortigen Deutschsprechenden, vermochte die tschechisch sprechende Majorität eigentlich gar nicht wahrzunehmen. „But the city proved essential to the transformation of his research profile by giving him the conceptual (and literal) space to detach himself from the pressures of the ever-growing quantum theory and enabling him to follow a hunch from several years earlier about the equivalence principle and gravitation." 32

Summa summarum: So wenig wie die allerdings sehr prosaische zeitgenössische Gegenwart in Athen den Mythos dieser Stadt entkräften kann, erging es Einstein mit seinem magischen Prag, dieser golden gesegneten Stadt der Astronomie und Astrologie, die zudem in den damaligen Tagen das WeltZentrum bedeutender epistomologischer Überlegungen darstellte. Wie auch in Wien, wurden in Einsteins damaligem Prag Gedankengängen geboren, die ihn zentral angingen. Ihn, dieses Physikgenie mindestens des 20. Jahrhunderts, das seine weltumstürzende Theorie einst in Bern inmitten einer transdisziplinären Diskussionsrunde entwickelt hatte, betraf die „Wiener Schule“ durchaus, ihn, der sich eingehend und wie schon referiert, - auch mit dem Don Quichotte beschäftigt hatte. Albert Einstein hat diesen Roman nie vergessen. Er war ihm gewiss ein Maßstab für seine Beurteilung der Brod'schen und Kafka'schen Texte. Don Quichotte war schließlich paradoxal angelegt, und eben dieses Merkmal galt gleichermassen für die einschlägigen epistomologischen Theorien der sog. „Wiener Schule“ (die eigentlich „Prager Schule“ heißen sollte, war sie doch eher in Prag, als in Wien zu Hause gewesen). Hier sind Namen aufzuzählen, bekannte, bedeutende wie: Bernard Bolzano, Ernst Mach, Franz Brentano, Rudolf Carnap, Moritz Schlick, Edmund Husserl, Karl Popper, Friedrich von Hayeck, Paul

\footnotetext{
${ }^{32}$ Michael D. Gordin, Einstein in Bohemia, a. a. O., S. 75.
} 
Feyerabend; wobei diese Aufzählung so wenig vollständig erscheint, wie sie alphabetisch ausgefallen ist. (Thomas Kuhns berühmter „Paradigmawechsel“, bereits mehrfach angeführt und seinerseits in den 1960er Jahren formuliert, gehört selbst in die Nachfolge des obigen „Wiener Kreises“). Also: Was vermag die unzureichende Gegenwart gegen den vollkommen scheinenden Mythos? Zählte das heutige, oder eigentlich das vergangene Prag? Wieso bedeutete der Prag-Aufenthalt für Einstein so viel, und mit zunehmendem Alter immer nur noch mehr? Insgesamt kann man die Summe aus Einsteins in Prag erlangten Erkenntnissen wohl wie folgt ziehen: "It had not been a great success in terms of leading directly to a full-blown theory of general relativity, but it had provided a passage out of the quantum rut and set him on a course to one of the most

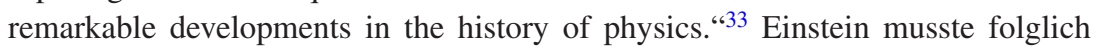
zufrieden sein mit den Ergebnissen seiner Prager Professur - bis hin zu seinem spektakulären Geigen-Auftritt im Zusammenhang mit der erwarteten Verleihung des Nobelpreises, worüber nun zu berichten sein wird.

Hatte der Prager „genius loci“ den Zürcher inspiriert, vermeinte Einstein in Prag eine solche „Be-geisterung“ zu verspüren, wie sie sozusagen nur aus den Tiefen des dortigen, mit Wissenschafts- und Kulturgeschichte gedüngtem Boden aufzusteigen vermochte? Sein ältester Sohn Hans Albert sprach noch im Jahr 1970 sich eindeutig für diese Annahme aus. „If they say that my father was not enthusiastic about Prague, they are greatly misled. He loved Prague and he spoke about it with enthusiasm even many years later.“34 Das gilt ungeachtet aller herabsetzender Bemerkungen Einsteins über das damals gegenwärtige Prag, über Kollegen und Studenten - die sich ebenfalls zitieren lassen, den aufgeklärten Zürcher Lokalpatrioten in Einstein demonstrierend: „Much misery alongside boasting and arrogance. Class prejudice. Little true cultivation (Bildung). Everything is byzantine and priest-ridden". ${ }^{35}$ Dagegen steht, was Gordin mit dem Recht des objektiven Geschichtsschreibers eben auch hat festhalten können: „The physicist was happy with his work on general relativity, even though he eventually abandoned the static theory. He came to appreciate the status of an ordinary professor, and he made good friends. " ${ }^{36}$ Solch eudämonistisches Dasein

\footnotetext{
${ }^{33}$ Michael D. Gordin, Einstein in Bohemia, a. a. O., S. 79.

${ }^{34}$ Rudolf Kolomy, Albert Einstein v Praze, Vesmir 53, no. 4, 1974, S. 113.

${ }^{35}$ An Alfred und Clara Stern, 17. März 1912, zitiert nach Michael D. Gordin, Einstein in Bohemia, a. a. O., S. 87.

${ }^{36}$ Michael D. Gordin, Einstein in Bohemia, a. a. O., S. 105.
} 
hatte dazu beigetragen, dass Einstein seinen Tribut in Form von öffentlichen Vorträgen stets und willig entrichtete. So etwa auch an jenem 23. Mai im Jahr 1911, wo er vor Brod, Hopf und Kafka über seine „Spezielle Relativitätstheorie“ sprach. Ein öffentliches Ereignis, wie es nicht nur in der Bohemia angezeigt wurde. Der Geigenspieler Einstein war schon zuvor häufiger Gast in Berta Fantas Salon gewesen, direkt über der bekannten Apotheke gelegen, dem „Einhorn“ des Fanta-Ehemannes Max. Am „Alten Marktplatz“, in Prags historischem Zentrum situiert, unweit der weltbekannten astronomischen Uhr. „Einstein did not simply arrive in Prague, work on general relativity, and then leave. On the contrary, he participated quite actively in the various forms of bourgeois sociability that where typical of that time and place." 37 Von hier ging nicht nur die bereits beschriebene Bekanntschaft Kafkas mit den Einstein'schen Theorien aus. Sie wurde dann am 24. Mai 1912 erneuert im Kreis von Hopf, Robert Weltsch und Kafka, in Gesprächen über Freud und Jung, wie wiederum Brod in seinem (dann leider passagenweise vernichteten) Tagebuch es sich notiert hat. ${ }^{38}$

Auch diese enge gesellschaftliche Einbindung führte $\mathrm{zu}$ einem weiteren Besuch Einsteins in Prag, der uns nun interessieren soll. Am 6. Januar 1921, also ein rundes Jahrzehnt nach Einsteins erstem Aufenthalt in Prag, stand Philipp Frank, der kommende Biograph Einsteins (und Nachfolger desselben auf dessen Prager Lehrstuhl, dabei seinerseits Philosoph wie Physiker in einem, wie dann auch Werner Heisenberg, oder noch später Stephen Hawkings) auf dem Bahnsteig von Prags Hauptbahnhof. Der Nachfolger auf Einsteins „Lehrkanzel“ wartete hier auf seinen Meister, das Objekt seiner biographischen Begierde. Der Bahnhof hieß jetzt schon nicht mehr nach dem ewigen Kaiser des Habsburger Reichs „Franz Josef Station“; sondern nunmehr knapp Wilson Station, nach jenem US-amerikanischen Präsidenten benannt, der die neue Unabhängigkeit der Tschechoslovakei in Versailles mit durchgesetzt hatte. Der kommende Biograph seinerseits arbeitete an der Universität immer noch im gleichen Raum, wie, eine Dekade vor ihm, Albert Einstein. Doch ansonsten waren die politischen Verhältnisse grundlegend umgestürzt worden, Resultat des von Österreich und Deutschland katastrophal verlorenen „Großen Krieges“. Auch Einsteins Status erschien einschneidend verändert, freilich mit konträrem Vektor. Seit dem Jahr 1919 lag der unabweisbare, weil empirische Beweis vor, dass die Einstein'schen Theorien

\footnotetext{
${ }^{37}$ Michael D. Gordin, Einstein in Bohemia, a. a. O., S. 92.

${ }^{38}$ Margarita Pazi, Franz Kafka, Max Brod und der ,Prager Kreis ‘, in: Karl Egon Grözinger, Franz Kafka und das Judentum, Frankfurt 1987, S. 88.
} 
richtig waren. $\mathrm{Zu}$ diesem Zeitpunkt war nicht lediglich $\mathrm{zu}$ erwarten, sondern man wusste damals bereits, dass der Nobelpreis auf Albert Einstein zukommen würde. Zurück zur Prager Szenerie, zur Wilson Station: Das Genie erschien in der Waggontür; strubbelig, irgendwie südländisch-zigeunerhaft sah es aus, war übernächtig und samt seiner Violine eingetroffen. Sein Nachfolger und Biograph in spe erwartete ihn. Sah buchstäblich vom Perron zu ihm auf. „The man he was searching for descended from the train , and still looking like an itinarent violin virtuoso", he recalled." ${ }^{39}$ Der zigeunerartige Geiger war allerdings gleichzeitig der kommende Nobel-Preisträger. Aber erst im Herbst sollte dies feststehen, nachdem Einstein, wissend, was kommen würde, dennoch durch den Fernen Osten gereist war, und so die Vergabe des Nobelpreises beinahe noch ruiniert hatte durch sein hartnäckig-bohemisch-weltbürgerliches Festhalten an der so geliebten Schweizer, nein: Züricher Staatsbürgerschaft. Jedenfalls: Als ein zigeunerhafter Geigenvirtuose betrat dieser Mann, der Nachfolger von Ptolemaeus, Kopernikus und Newton, womöglich das größte Physik-Genie, das die Menschheit überhaupt hervorgebracht hatte, erneut den mythischen Boden der Moldaumetropole. Als ein siegreicher Geiger würde er ihn wieder verlassen, der Nobelpreisverleihung entgegenzugehen, und nach einem furiosen Sieg der musischen, und wenn man so will: Jüdisch-assimilierten Intelligenz über die platte Dummheit eines bereits heraufziehenden, neuen, aber auch seinerseits physikalisch-technisch interessierten Barbarentums. Dessen Parteigänger gab es damals nicht nur in der Weimarer Republik. Sondern auch in Prag als der noch neuen Metropole des nun selbstständigen tschechoslovakischen Staates; sie waren ihrerseits an der damals noch bestehenden „Deutschen Universität“ zu Hause. Ihr Anführer war ein gewisser Oskar Kraus, Professor der Philosophie und borniert-eloquenter Vertreter einer ,arischen“ Physik. Dieser Kraus benutzte die Gelegenheit, in der Diskussion nach Einsteins öffentlichem Vortrag, ,den Juden zu stellen“. Er attackierte den zigeunerhaften Einstein als den Vertreter einer ,jüdischen Physik“, nachdem dieser, übrigens, zuvörderst über die Lichtgeschwindigkeit als eine unerreichbare Größe gesprochen hatte, mit einer gekonnt ,witty and cutting broadside against the fundamental postulates of relative motion." 40 Jetzt aber ereignete sich das Unglaubliche, das damals sogar Oskar Kraus zum Lachen gebracht haben muß, wie immer die denkwürdige Episode in Wahrheit seine blamable Niederlage beinhaltete. In der

\footnotetext{
${ }^{39}$ Philipp Frank, Einstein. His Life and Times, Cambridge 2002, S. 170.

${ }^{40}$ Michael D. Corbin, Einstein in Bohemia, a. a. O., S. 110.
} 
Form eines epochemachenden „happenings“ kamen jetzt die transdisziplinären Elemente einer „Dritten Kultur“ in Gestalt von Physik, lässig abgewehrtem, darin „Zürcher“ Anti-Antisemitismus und - eben der Musik zusammen. Die Wahrheit schien auf in Form einer Mozart-Sonate. Gegen die Finsternis eines neuen, rabiaten Obskurantismus in Sachen Wissenschaft wie Mitmenschlichkeit gleichermaßen gestellt - und das in Kafkas Prag. In einem Prag, das damals immer noch die Stadt der astronomischen Erkenntnis war - und gleichermaßen bereits die finsterer antisemitischer Pogrome. Das Licht des göttlichen Mozart, in Prag seit Mörikes Mozart auf der Reise nach Prag an seinem angestammten Platz, wurde nun vom vermutlich größten Physiker der Menschheitsgeschichte entzündet, und vernichtete die Mächte der heraufziehenden neuen Finsternis, noch einmal, aber leider nicht für immer. ${ }^{41}$ Zur Verblüffung aller Anwesenden, zur Erheiterung selbst des Stellvertreters eines sich neu durchsetzenden absoluten Bösen, weigerte sich Einstein, auf die wissenschaftlichen Obskuritäten seines Diskussionspartners überhaupt, will sagen: Verbal zu antworten. Stattdessen machte der Mann wahr, was seinem ungarisch-balkanischen Phänotyp entsprach (und was Kraus, als den vermutlich glatt gekämmten Vertreter der ,arischen Rasse", nur befeuert haben kann): Statt sich zu rechtfertigen, womöglich mit den Mitteln der Mathematik, griff dieser strubblige Mann zur Geige. Und spielte, lange und intensiv, mit dem überirdischen Genuss des Mozart-addics, eudämonistisch musikversunken in aller Hitze des ideologisch so aufgeladenen Disputs, eine der Geigen-Sonaten des „göttlichen Kindes“! Welch ein tableau; von Leben selbst angerichtet, und vom „itinarent violin virtuoso“ Einstein mit schauspielerischer verve ausgeführt. Eine Sternstunde der Menschheit.

Und zudem eine, die am rechten Platz sich ereignete. Zu diesem Komplex existieren Überlegungen, die heranzuziehen an dieser Stelle weiterführend erscheint. Zumal sie, partiell aus gleichem altösterreichischen Kulturraum stammend und ebenfalls die Annahme einer "Magie des Ortes“ favorisierend, wissenschaftliche Dignität aufzuweisen haben, wenn sie auch weniger musikalisch als das erscheinen, was als Albert Einsteins aparter ViolinenKonter geschildert werden konnte. Sigmund Freuds kulturpessimistischer Aufsatz von 1930 Das Unbehagen in der Kultur verglich das kollektiv Unbewußte mit einer Stadt, in der alle Schichten der kulturhistorischen Entwicklung noch

\footnotetext{
${ }^{41}$ Hier sei daran erinnert, dass der Haupt-Einwand gegen unsere Darstellung darin bestand, daß Musik wie auch Einsteins Physik darin nur „spekulativ“ einbezogen wären. Wenn man die Geige zu spielen wüsste à la Einstein, wäre dann ein Mittel zur Hand, die Dämonen auszutreiben?
} 
gleichzeitig vorhanden sind. Also auch räumlich noch koexistieren - sozusagen eine Computersimulation von zeitlich aufeinander folgenden, dann archäologisch übereinander gelegten Entwicklungsschichten des Gemeinwesens. Die Freud'sche Vorstellung beruhte auf einer Grundannahme der Psychoanalyse, die wir, siehe Kafkas mehrfache Berufungen auf Freud, mit Fug zum Kafka'schen Gedankenfundus rechnen können. Dass die Phylogenese die Ontogenese vorbildet, gehört in diesen Gedankenkomplex ebenso hinein, wie die Überzeugung, dass die spezifischen Gedächtnisspuren eines besonderen Ortes, über Generationen überliefert, das Individuum prägen, jedenfalls beeinflussen werden. $\mathrm{Zu}$ verweisen wäre an dieser Stelle auch auf die entsprechenden Überlegungen des Selbstbiographen Canetti, der, unter anderem, auch ein Wiener gewesen ist, und der diese Thematik in Die gerettete Zunge facettiert traktiert hat. Freud schrieb: „Seitdem wir den Irrtum überwunden haben, dass das uns geläufige Vergessen eine Zerstörung der Gedächtnisspur, also eine Vernichtung bedeutet, neigen wir zu der entgegengesetzten Annahme, dass im Seelenleben nichts, was einmal gebildet wurde, untergehen kann, dass alles irgendwie erhalten bleibt und unter geeigneten Umständen, z. B. durch eine weit reichende Regression, wieder zum Vorschein gebracht werden kann. “42 Dass alle Kunst, als gesellschaftlich geduldete Form einer Neurose, auch notwendig die Züge einer Regression tragen muss, gemäß der berühmt gewordenen Freud'schen Formel von der gesellschaftlich erlaubten Reinfantilisierung des Kunstschaffenden, versteht sich in diesem Zusammenhang von selbst. Weiterhin Freud: „Nun machen wir die phantastische Annahme, Rom sei nicht eine menschliche Wohnstätte, sondern ein psychisches Wesen von ähnlich langer und reichhaltiger Vergangenheit, in dem also nichts, was einmal zustande gekommen war, untergegangen ist, in dem neben der letzten Entwicklungsphase auch alle früheren noch fortbestehen." ${ }^{43}$ Wie Freud weiter darlegte, würde das für das heutige Rom bedeuten, dass auf dem Palatin weiterhin die Kaiserpaläste und das Septizonium des Septimus Severus sich erhöben. Und dass die Engelsburg noch die nämlichen Zinnen trüge, die sie zur Zeit der Gotenbelagerung schmückten. Machen wir nun die überhaupt nicht phantastische literaturtheoretische Annahme, dass man in dem Schreiber Kafka alle jene Spuren sozialer Energie wieder- und wirksam finden könnte, die Prag während seiner Lebenszeit durchzogen, gewinnen wir womöglich einen weiteren „Schlüssel“ zu Kafkas Literatur. Der käme dann aus Wien.

\footnotetext{
${ }^{42}$ Sigmund Freud, Das Unbehagen in der Kultur, in: Gesammelte Werke, hrsg. v. Alexander Mitscherlich, Frankfurt/Main 1982, Bd. IX, S. 201.

${ }^{43}$ Sigmund Freud, Das Unbehagen in der Kultur, a. a. O., S. 201.
} 
Das Gedankenexperiment würde nicht nur dem Verschollenen gelten. Auch Kafkas Prag im Process wäre dann als jene Moldaumetropole wiederzuerkennen, die in außerordentlichem Umfang von sozialen, aber bereits auch rassentheoretisch begründeten Nationalitätenkämpfen bestimmt wurde. Wo also nationale Ängste mit übernationalen Hoffnungen widersprüchlich koexistierten, was bewirkte, dass diese Groß-Stadt der habsburgischen „Göttin der Gerechtigkeit" ebenso angehörte, wie noch der slavischen Gründer-Fürstin Libuša. Hoch entwickeltes formaljuristisches Denken steht neben noch archaisch-schamanenhaft, hetzerisch betriebener „Rechts"findung. Die „Göttin der Gerechtigkeit" erweist sich zugleich als „Göttin der Jagd“, wie Kafkas zweiter Roman es auf den Dachböden der Vorstädte zum „kafkaesk“ eindrücklichen Ereignis werden lässt. Der Sachverhalt mag für alle Städte und Herkunftslandschaften eines Dichters gelten. Aber er gilt eben in besonderem Maße, wenn die Herkunftsstätte zugleich der leidenschaftlich umkämpfte Existenzraum verschiedener ethnischer Gruppierungen gewesen ist. Es war Kafka, der zu Gustav Janouch über sich selbst und sein Innenleben in Wendungen gesprochen haben soll, die von geradezu struktureller Identität mit den oben zitierten Gedanken Freuds zeugen: „In uns leben noch immer die dunklen Winkel, geheimnisvollen Gänge, blinden Fenster, schmutzigen Höfe, lärmenden Kneipen und verschlossenen Gasthäuser. Wir gehen durch die breiten Straßen der neuerbauten Stadt. Doch unsere Blicke und Schritte sind unsicher. Innerlich zittern wir noch so wie in den alten Gassen des Elends ... Die ungesunde alte Judenstadt in uns ist viel wirklicher als die hygienische neue Stadt um uns. Wachend gehen wir durch einen Traum, selbst nur ein Spuk vergangener Zeit." ${ }^{\text {"4 }}$ In diesem Sinn also könnten wir in Kafka getrost einen „Freudianer“ erblicken. Es scheint zu gelten, dass gerade die „unheimlichen“ und noch „magischen“ Züge des Stadtmythos”, wie sie ihrerseits die Topographie der Stadt an der Moldau geprägt haben, in Kafkas Literatur den Weg in die Zeichenwelt der Schrift, und damit in die Welt des dichterischen logos, gefunden haben. Wiederum Aleida Assmann hat die Frage aufgenommen, die vor ihr Freud gestellt hatte, indem sie Geoffrey Hartman aus Saving the Text zitierte: „Wenn wir sowohl die Stimme wie den Anblick vergessen, oder ihnen erlauben, als bloß phänomenologisch beiseite zu treten, was bleibt? Was verbleibt, Substanz, Felsen, eine Gründung, ein Haus oder ein Pfad?“45 Mit Recht

\footnotetext{
${ }^{44}$ Gustav Janouch, Gespräche mit Kafka, Frankfurt/Main 1968, S. 134.

${ }^{45}$ Aleida Assmann, Erinnerungsräume, München 1999, S. 298: „If we elide both voice and look, or allow them to slip away as purely phenomenal, what is left? What ,demeure', substance, rock, foundation, house, path?".
} 
kann darauf verwiesen werden, dass die Formel vom „Gedächtnis der Orte“ es offenlässt, ob es sich um den genitivus subjektivus oder den genitivus objektivus handelt. Vermitteln die Orte denen, die an ihnen aufwachsen, eine besondere Art von Gedächtnis? Tragen sie womöglich selbst in ihren materiellen Substraten ein Gedächtnis mit sich, das sich denen mitteilt, die in ihnen wohnen, leben und arbeiten? Beschwört Kafkas irrational hüpfende Spule Odradek mit ihrer deutschtschechisch umkämpften Sprach-Herleitung nicht die gesamte Stadt Prag als den Ort von erbitterten Sprachkämpfen?46 "Auf den ersten Blick erkennt wohl jeder Sprachwissenschaftler in Kafkas literarischem Neologismus 'Odradek' einen Eigen- oder Familiennamen. Die Ursache dafür liegt in dem diminutiven, patronymischen Suffix, das für zahlreiche Eigen- und Familiennamen in den slawischen Sprachen und vor allem dem Tschechischen kennzeichnend ist." "47 „Odradek“ also als eine spezifische Chiffre jenes Prag, das zu Kafkas Zeiten zwar immer noch das alte „magische“ war, aber nun aktuell bestimmt von den Nationalitätenkämpfen des anbrechenden nationalistisch-faschistischen 20. Jahrhunderts. Die Charakteristik des Orts zusammengefasst in einer rätselhaften hüpfenden, womöglich elektromagnetisch bewegten Spule namens „Odradek“, dieser Frucht vom gleichen „kafkaesken“ Stamm wie die hüpfenden Bälle des Junggesellen Blumfeld.

Bereits Cicero hat, im Rahmen der Ausarbeitung einer spezifisch römischen Mnemotechnik, die „Kraft der Erinnerung“ gerühmt, wie sie insonderheit von den Orten ausgehen kann. Assmann andererseits meint, dass bereits bei dem antiken Rhetor der Übergang von den „Örtern des Gedächtnisses“ zu den „Erinnerungsorten" angelegt und also bereits damals ein Denkbild entstanden sei, das in unserem Zusammenhang auf die Stadt Prag appliziert werden könnte. Dass dabei auf Goethe, Kafkas eines großes Vorbild, rekurriert wird, macht die Sache nur einleuchtender. Im Versuch der Begründung der Symboltheorie gelangte nämlich Goethe (in seinem Briefwechsel mit Schiller) zur Überzeugung, dass die Symbolkraft der Orte noch viel größer sei als die der Gegenstände. Diese Erkenntnis setzte sich interessanterweise gleichsam hinter dem Rücken des Weimarer Klassikers durch: Als er, gegenüber Schiller, für seine Symboltheorie als Rückgrat aller modernen Dichtung argumentierend, spontan darauf verfiel, gleich zwei - Orte zu benennen. „Bis jetzt habe ich nur zwei solcher Gegen-

\footnotetext{
${ }^{46}$ Vgl. dazu Gernot Wimmer. Kafka zwischen Judentum und Christentum, Würzburg 2012, darin S. 165 ff.: Tomislav Zelić, Odradek oder Das Enigma der Identität.

${ }^{47}$ In: Gernot Wimmer, a. a. O., S. 165.
} 
stände gefunden: den Platz auf dem ich wohne, der in Absicht seiner Lage und alles dessen was darauf vorgeht in jedem Momente symbolisch ist, und den Raum meines großväterlichen Hauses, Hofes und Gartens, der aus dem beschränktesten, patriarchalischen Zustande, in welchem der alte Schultheiß von Frankfurt lebte, durch klug unternehmende Menschen zum nützlichsten Waren- und Marktplatz verändert wurde.“48 Die Orte in ihrem städtischen Wandel erscheinen bei Goethe als der Sitz eines besonderen Gedächtnisses. Sind ihm in seinem Frankfurt so gegenwärtig, wie es im Fall Kafkas der innere Ring-Bezirk seines Prag gewesen war. Wie Freud ist Goethe der Auffassung, dass man an den Orten in die Tiefe gehen muss, will man ihres symbolschaffenden „genius loci“ habhaft werden. Denn die Räume in ihrer Horizontalen sind allesamt, selbst zu Goethes Zeiten, bereits erforscht, kartografiert, mithin „er-schlossen“. Die „Vermessung der Welt" war bereits damals beendet, Humboldt an nahezu jedem Ort der Erde gewesen, wenn auch nicht in Asien, Australien, der Antarktis.. Nur in der Tiefe liegt seitdem noch das Geheimnis beschlossen, das über die symbolisch prägende Kraft der Ursprungsorte Auskunft zu geben vermöchte. Ein originär frühromantischer Gedanke, übrigens; Novalis, Schlegel, Tieck hätte er sofort eingeleuchtet. „Während ,Raum` zu einer ... Kategorie der ... Disponibilität geworden ist, richtet sich die Aufmerksamkeit auf den ,Ort' mit seiner geheimnisvollen, unspezifischen Bedeutsamkeit. Dieses Geheimnis, das bestimmten Orten innewohnt, will (Goethe, B.N.) entbergen und, wie Silber aus den Bergwerken, als Beute davontragen." ${ }^{49}$ Ein solches Verfahren gleicht übrigens dem des „Perlentauchens“ nach sprechenden, Evidenz evozierenden Zitaten, die als neu zum Tönen gebrachte „Stimmen“ die Räume in ihrer symbolischen Dimension zum Sprechen bringen können, - ganz so, wie Hannah Arendt dieses Verfahren mit Rekurs auf Shakespeares Sturm (und auf Brecht/Benjamins Traum von einem Buch, das nur aus Zitaten bestehen und derart die Vergangenheit neu zum Sprechen zu bringen vermöchte) erörtert hat. Was wiederum seine Nähe zur eingangs herangezogenen Geertz'schen „Thick Discription“ besitzt. Die besonderen sozialen Energien, die Kafkas Prag ihrerseits „electrisch“ durchzogen, werden auf diese Art und Weise zu den „Augen des toten Vaters“. Welche, auf dem Meeresgrund als dem Raum aufbewahrter Vergangenheit abgelagert, bei dem englischen Dramatiker zu jenen „Perlen“ werden, die es nach Arendts und

\footnotetext{
${ }^{48}$ Johann Wolfgang Goethe, Briefe, in: Hamburger Ausgabe, hrsg. v. Erich Trunz, dtv Verlagsgesellschaft 1998, S. 298.

${ }^{49}$ Aleida Assmann, Erinnerungsräume, a. a. O., S. 300.
} 
Benjamins Erkenntnis zu bergen gilt. Ein Verfahren, das die Rekonstruktion des Vergangenen, ,so wie es einst wirklich gewesen“, in der Tat anstrebt, - aber nicht mehr naiv genug ist, an dessen vollständigen Erfolg zu glauben. Mithin modern und traditionell in einem, Provinz und Zentrum ebenso vermittelnd, wie andererseits die Substanz der Tradition mit der erkenntniskritischen Potenz der Moderne.

Vor allem erlaubte die heuristische Gleichsetzung von Stadttopographie und Archäologie des Unbewußten, gerade weil sie um ihre eigene Zeichenhaftigkeit weiß und immerhin von Goethe, Freud, Arendt und Assmann ausdifferenziert betrieben wurde, es wesentlich besser, die besondere Bedeutung der sogenannten „Durchhäuser“ zu verstehen, die für Prags Zentrum so charakteristisch waren - wie sonst wohl für keine andere Metropole der Welt. Egon Erwin Kisch hat betont, ,dass man durch ganze Stadtteile Prags gehen (kann), ohne die offene Straße als zu etwas anderem als zum bloßen Überschreiten benützen zu müssen, sozusagen auf dem Landwege." 50 Der Vergleich mit Paris (oder mit Wien) scheint hier dennoch nicht schlüssig: Denn im genauen Gegensatz zu Paris ist Prag niemals jener rationalen Umgestaltung, die den Truppen freieres Schussfeld schaffen sollte, unterzogen worden, wie die französische Hauptstadt durch die nachrevolutionäre Stadtplanung George Eugène Hausmanns. Auch das platzschaffende Schleifen der Stadtbefestigung hat Prag, anders als die Schwesterstadt Wien, so nie erlebt. Insofern gliche Paris (wenn denn Paul Valéry Recht hat mit seinem Vergleich zwischen dem Bild einer Stadt und dem menschlichen Gehirn, gezogen in seinem Essay Présence de Paris) dem Gehirn der Aufklärung, - was gerade auf Prag mit seinem Golem-Mythos und seiner Ghetto-Tradition so ganz und gar nicht zutraf. Wien mit seinem abgerissenen Ringwall scheint darin näher an Paris als an Prag zu sein, wie immer Wien und Prag durch Jahrhunderte zusammengehörten. Die Prager Durchhäuser glichen dann eher jenen Höhlenformationen im Unterbewusstsein, in denen das Alte und Verdrängte sich ablagert, ohne damit aus der Welt verschwunden zu sein. In diesen Durchhäusern lag jedenfalls die eigentliche Welt des Prager Bürgers Franz Kafka beschlossen. Das gilt im strengen topographischen Sinn ebenso, wie mentalitätsgeschichtlich, und auch vom Geist der Kafka'schen Literatur her. Was sich in diesem Bereich beobachten lässt, erhebt die Prägung des Kafka'schen Unterbewusstseins durch die Prager Geschichte zur Anschaulichkeit des metaphorischen logos in jener Literatur, die der Prager dann geschrieben hat.

${ }^{50}$ Zitiert bei Peter-André Alt, Franz Kafka. Der ewige Sohn, a. a. O., S. 44. 
Hier ist der Ort, einen weiteren Roman zu erwähnen, der für das internationale Kafka-Verständnis von nachhaltiger Bedeutung geworden ist: Nämlich Gustav Meyrinks 1915 erschienenen Der Golem. Dieser expressionistische Esoterik-Thriller, ein Sensationserfolg über die Jahre hinweg, hat das Bild des in Durchhäusern verborgenen und von vergessenen Gängen untertunnelten, noch altösterreichischen, zugleich aber bereits höchst „geheimnisvollen Prag“ geprägt, wie sonst kaum ein anderer. Meyrinks Roman zeichnete das Bild einer von ganz und gar abgründigen sozialen Energien durchzogenen Stadt, deren geheimnisvolle bis verbrecherische Dynamik auf das ehemalige Judenviertel konzentriert erscheint. Dort soll ja auch der sagenhafte Golem des Rabbi Löw sein Wesen getrieben haben (und erörtert worden sein oben auf dem Hradschin, am Hof des dort residierenden Habsburgischen Kaisers Rudolf). Die morbide Sexualität, über die selbstverständlich auch Meyrinks Prag verfügt, erscheint ihrerseits in Kafkas Process zur Stelle, gerade in ihrer Konnotation mit schmutzigem Kinder-Sex (was in neuerer Zeit manchen ostjüdischen Deuter dazu befeuert hat, Kafka als Kinderschänder zu verdächtigen, was der Mann aber gar nicht war. Auch Einsteins „Perversitäts“-Urteil besitzt darin seine Stütze, zielte aber wohl vor allem auf Frieda und K. im Ausschank des Schloss-Gasthofs, ihren Koitus in Bierpfützen). Gustav Meyrinks Roman verdankt seine Popularität vor allem der Tatsache, dass in ihm die alte Welt der Judenstadt von vor 1852 aufbewahrt wird, eine, wie man sehen wird, durchaus zwiespältige Erinnerung. Das alte Ghetto, die sogenannte ,Judenstadt“" war in den Jahren nach 1852 ,assaniert“, durchgehend und bis ins Verschwinden hinein modernisiert worden. Zugrunde lag dem das Toleranzedikt des Maria-Theresia-Sohnes Joseph, auf dessen Grundlage (und diesem katholisch-aufgeklärten Kaiser zu Ehren) nun ein ganz neues Viertel, „Josephsstadt“ oder „Josefov“ genannt, entstanden war. Allein sechs hundert private Häuser waren damals niedergerissen worden, die hergebrachte Struktur radikal entfernt worden bis auf das Jüdische Rathaus, die zentrale Synagoge, und den noch heute erhaltenen Friedhof. Weite Boulevards nach Pariser Muster durchzogen nun das Viertel, dessen Hauptachse die Tschechen deshalb die „Pariserin“ nannten. „By the time Einstein arrived in the city, Josefov was gone, memorialised in Gustav Meyrinck's gothic novel The Golem (19131915), set during the asanace. Einstein's engagement with Prague's Jews would happen outside of Josefov" ${ }^{51}$ aber das Bild der alten Judenstadt lebte weiter in Meyrinks Bestseller. Vor allem aber gerät hier die multinationale Kampfstadt

\footnotetext{
${ }^{51}$ Michael D. Gordin, Einstein in Bohemia, a. a. O., S. 191.
} 
Prag ins Bild, in der man einander mit dem mitleidslosen Blick des Rassenideologen zu fixieren pflegte. Das sensationalistische Buch lebte ganz eigentlich von der riskanten Mischung zwischen Schmuddelsex und Rassenkampf. „Mich ekelte vor ihrem zudringlichen Lächeln ... Sie muss schwammiges, weißes Fleisch haben wie der Axolotl, den ich vorhin im Salamanderkäfig bei dem Vogelhändler gesehen habe ... Unter den Judengesichtern, die ich Tag für Tag in der Hahnpassgasse auftauchen sehe, kann ich deutlich verschiedene Stämme unterscheiden, die sich so wenig durch die nahe Verwandtschaft der einzelnen Individuen verwischen lassen, wie sich Öl und Wasser vermengen wird ... Diese Stämme hegen einen heimlichen Ekel und Abscheu voreinander, der sogar die Schranken der engen Blutsverwandtschaft durchbricht ... Rosina ist von jenem Stamme, dessen rothaariger Typ noch abstoßender ist als die anderen. Dessen Männer engbrüstig sind und lange Hühnerhälse haben mit vorstehenden Adamsäpfeln."52 Das zitiert man nicht gern. Dennoch liegt hier ein sehr aussagekräftiges und darum wichtiges, eben deshalb zitierenswertes Zitat gerade im Sinne von Geertz' ethnologischer Methode vor, das politischer Korrektheit auf keinen Fall zum Opfer fallen darf. Meyrinks Hass- und Kampfszenen peitschten die Emotionen des Lesers hoch und lebten durchaus von der Atmosphäre eines Rassenkampfes, der seinerseits dann auch in Kafkas Process gegenwärtig sein wird, und der in Dialogen wie dem folgenden seinen Ausdruck findet: „Ich hasse, genaugenommen, auch gar nicht ihn. Ich hasse sein Blut. Verstehen Sie das? Ich wittere wie ein wildes Tier, wenn auch nur ein Tropfen von seinem Blut in den Adern eines Menschen fließt ..." ${ }^{43} \mathrm{Zu}$ diesen Orgien eines hemmungslos sozialdarwinistischen Kampfes fügt sich die Kulisse des abgründigen Prag, in dem alle Häuser, samt der „Durchhäuser“, ihr satanisches Eigenleben gewinnen. „Dort ein halbes, schiefwinkliges Haus mit zurückspringender Stirn, - ein andres daneben: vorstehend wie ein Eckzahn. Unter dem trüben Himmel sahen sie aus, als lägen sie im Schlaf, und man spürte nichts von dem tückischen, feindseligen Leben, das zuweilen von ihnen ausstrahlt, wenn der Nebel der Herbstabende in den Gassen liegt ... Oft träumte mir, ich hätte diese Häuser belauscht in ihrem spukhaften Treiben und mit angstvollem Staunen erfahren, dass sie die heimlichen Herren der Gassen seien, sich ihres Lebens und Fühlens entäußern und wieder an sich ziehen können - es tagsüber den Bewohnern, die hier hausen, borgen, um es in kommender Nacht mit Wucherzinsen wieder zurückzufordern. "54

\footnotetext{
${ }^{52}$ Gustav Meyrink, Der Golem, München/Wien (Langen Müller), 1979, S. 13.

${ }^{53}$ Gustav Meyrink, Der Golem, a. a. O., S. 132.

${ }^{54}$ Gustav Meyrink, Der Golem, a. a. O., S. 32.
} 
Der zitierten Stimme muss freilich ein charakteristisches Element des Kafka'schen Pragerlebnisses einschränkend entgegengestellt werden: Die zentrale Welt der Durchhäuser war dem Prager eher Schutz als Bedrohung. Die in der Kafka-Literatur beliebte Verkoppelung der Durchhäuser mit dem „Unheimlichen der Kafka'schen Romane“ ist eher ungenau, partiell sogar irreführend. Denn ebenso wie in Kafkas Erzählung Das Urteil, ein Beispiel zu greifen, das, laut Freud, ,arme Ich“ immer in Gefahr steht, von einem bedrohlichen Unterbewusstsein (gerade auch im Verbund mit dem Über-Ich) verschlungen zu werden, so gab andererseits die zentrale Welt der Durchhäuser um den Altstädter Ring herum jene vertraute und ,ich-nahe“ heimische Welt ab, die im Fall des jungen Franz Kafka von einem anderen und „wirkliche“ Ring des Bedrohlichen, nämlich von Prags Vorstädten, umgeben erschien. Deshalb wird das Charakteristikum der baumlosen Vorstädte im (zum Erscheinen von Meyrinks Roman zeitgleichen) Process darin liegen, dass Kafkas K. in den Vorstadt-Straßen allen feindlichen Blicken schutzlos ausgesetzt erscheint, deren Baumlosigkeit also mangelnden Schutz bedeutete. Hier herrscht ein erbarmungsloses Über-Ich, das im Es der stets feuchten und koitusbereiten Waschfrauen seine Ergänzung gefunden hat. Die zentrale Welt der „Durchhäuser“ dagegen bot Schutz vor solch Foucault'schen Blick-Attacken. Ihr Labyrinthisches wirkte eher beschützend, ganz so wie der „Bau“ des Tieres in Kafkas gleichnamiger später Erzählung. Deshalb beginnt der Process ja auch mit jener Szene, in der das Intimste, das Schlafzimmer, plötzlich öffentlich geworden ist; schockartig ausgesetzt jedem Nachbarsblick. Was Franz Kafka einmal gegenüber Friedrich Thierberger als seine Heimat-Welt umrissen hat, fiel denn auch bezeichnenderweise zusammen mit der vertrauten Innenstadt: „Hier war mein Gymnasium, dort in dem Gebäude, das herübersieht, die Universität und ein Stückchen weiter links hin, mein Büro. In diesem kleinen Kreis und mit dem Finger zog er ein paar kleine Kreise - ist mein ganzes Leben eingeschlossen. "55 In diesen Ring war Franz Kafkas Leben eingeschlossen - nicht nur beengend, sondern eben auch beschützend. Hier „sahen“ sogar die Gebäude freundlich „,herüber“. Eine im Wortsinn überschaubare Welt: „Lediglich Kafkas Büro, das Gebäude der ,Arbeiter-Unfall-Versicherungs-Anstalt für das Königreich Böhmen' am Pořič (Nr. 7) führte wenige Schritte aus der Altstadt heraus. Das Gymnasium war im Kinsky-Palais (Altstädter Ring 6) untergebracht (in dem sich später auch das väterliche Geschäft befand), die Universität im

\footnotetext{
${ }^{55}$ Nach Friedrich Thierberger, ohne Quellenangabe zitiert bei Hartmut Binder/ Pavel Patik, Kafka. Ein Leben in Prag, Essen/München 1993, S. 17.
} 
Klementinum, mit dem Kreuzherrenplatz an die Moldau und die Karlsbrücke grenzend. Die Hörsäale der juristischen Fakultät lagen im Karolinum, neben dem Deutschen Landestheater. Um den Altstädter Großen und Kleinen Ring gruppierte sich das Rathaus, die Niklaskirche (an die sich Kafkas Geburtshaus anlehnte), auf der Rückseite des Rathauses das Haus ,Minuta', und ein paar Schritte vom Kinsky-Palais und der Teinkirche das Oppeltsche Haus, in dem Kafkas Eltern später wohnten. Vom Altstädter Ring führte nach Osten die Zeltnergasse, im zweiten Haus links (Nr. 3) die Wohnung der Eltern während Kafkas letzten Gymnasialjahren und der Universitätszeit. Wieder ein wenig weiter (auf der rechten Seite, Nr. 12) das väterliche Geschäft der frühen Prager Jahre ..."56 Am Ende der Niklasstraße rechts, das Eckhaus (Nr. 36) mit dem Blick auf den Hradschin und das Belverdere, lag die elterliche Wohnung ab 1907. Neben der Niklasbrücke wurde die Moldau damals, östlich, von der FranzJosephs-Brücke, nach Süden zu vom Kettensteg, der Karlsbrücke, der KaiserFranz-Brücke und der Palackybrücke überspannt. Die Karlsbrücke, „... nach ihrem Einsturz 1890 wieder neuerrichtet, war übrigens diejenige, für deren Überschreiten keine Maut erhoben wurde - eine Sitte, die sich bei den anderen Brücken zäh bis ins Jahr 1918 hielt. " ${ }^{\text {57 }}$ Nicht dieses historisch gewachsene (und bis zuletzt deutsch bestimmte) Prager Zentrum bedrohte damals Franz Kafka, und fixierte ihn mit feindlichen Vorstadt-Blicken. Sondern ein sich ausdehnendes anonymes Etwas, das den Ich-nahen, vertrauten und Schutz bietenden Bereich der schützend überdachten Innenstadt zu verschlingen drohte: Jene zumeist baumlos offenen Vorstädte, die zu Kafkas Lebzeiten aufgrund der damaligen Industrialisierung dramatisch anwuchsen (und die historische Innenstadt bereits um die Jahrhundertwende optisch wie demographisch überwältigen sollten). Wie im Blickzugriff des (tschechischen) Nachbarn auf K.'s Schlafzimmer, machte schon zuvor der Zugriff der Vorstädte aufs Zentrum aus Prag eine tschechische Stadt - ganz so, wie der Zugriff der normalerweise peripheren psychischen Instanzen Es und Über-Ich auf das zentrale Ich (im Urteil etwa), seinerseits zum Todesurteil für das Individuum zu geraten vermag. Der Held im Urteil jedenfalls springt in den Fluss, von einer Brücke, über die im gleichen Augenblick ein „,unendlicher Verkehr" hingeht. Auch Michel Foucaults Herrschafts-Blick als Zeichen moderner Rationalisierung der Macht ist hier nicht weit entfernt - und dann im Schloss wiederzufinden. Die Dialektik von Zentrum und Provinz bewährt sich darin, und sie ist nicht zufällig besonders intensiv im Weltkriegs-Roman Der

\footnotetext{
${ }^{56}$ Klaus Wagenbach, Franz Kafka, a. a. O., S. 68.

${ }^{57}$ Klaus Wagenbach, Franz Kafka, a. a. O., S. 68.
} 
Process zu Hause. Über das bedrohliche Außen, das gespenstische Etwas jenseits seiner vom Ich beherrschten Erfahrungswelt, hat Kafka selbst gegenüber dem Schwager Karl Hermann einmal einsichtsvoll gesagt, ,er hätte es stets mit einem gemischten Gefühl von Angst, von Verlassensein, von Mitleid, von Hochmuth, von Reisefreude, von Männlichkeit“"58 durchstreift. Dieses bedrohliche Andere, in dem er zwar ansatzweise ein zweiter Odysseus, doch wesentlich ein verhinderter Fabrikgründer ${ }^{59}$ (und somit gar kein Entdecker) gewesen ist, hat Kafka nur als die Infragestellung seiner eigenen Existenz wahrnehmen können. In den Prager Vorstädten ging Unkontrollierbares vor sich - unter anderem in der Fruchtbarkeit der dort beheimateten, verlockenden „Waschfrauen“, Fabrikarbeiterinnen und die Küche besorgenden Eheweibern, denen der Angeklagte K. im Process verfällt. Vor allem aber war dort eine bedrohliche Affektivität und aggressive Stupidität der massenhaft versammelten Kleinbürger und Proletarier in Erfahrung zu bringen, in Kafkas Schilderung ganz überwiegend Tschechen. In dieser Situation beschreibt Kafka eine Erfahrung, die Elias Canetti in Masse und Macht breit analysiert hat. Sie gab nicht zufällig das Lebensthema dieses im Habsburgerreich geborenen, in seinen Vorfahren ehemals spanischen Juden ab. In den Vorstädten, also im Ich-fernen Bereich, gebar der (tschechische) Antisemitismus, der nirgendwo anders so blühend heranwuchs, für den Autor Kafka ganz ähnliche Geschöpfe, wie sie auf den Zeichnungen des Pragers Alfred Kubin zu sehen sind, beim Betrachter die pure Angst evozierend. Dieses Massen-Geschöpf der Vorstädte, das genaue Gegenbild der individualistischen, reflektierten, vereinzelten, affektgehemmten und durchweg intellektuell gut ausgestatteten und ausgebildeten K.'s in Kafkas Literatur lässt sich mit der Stimme Freuds aus seiner Arbeit Massenpsychologie und Ich-Analyse von 1921 zur Anschaulichkeit bringen: „Wir sind von der Grundtatsache ausgegangen, dass ein Einzelner innerhalb einer Masse durch den Einfluss derselben eine oft tiefgreifende Veränderung seiner seelischen Tätigkeit erfährt. Seine Affektivität wird außerordentlich gesteigert, seine intellektuelle Leistung merklich eingeschränkt; ... ein Erfolg, der nur durch die Aufhebung der jedem Einzelnen eigentümlichen Triebhemmungen und durch den Verzicht auf die ihm besondere Ausgestaltung seiner Neigungen erreicht werden kann." ${ }^{60}$ Nicht nur im Falle Freuds, sondern auch in dem Canettis:

\footnotetext{
${ }^{58}$ Franz Kafka, Tagebücher 1909-1912, Bd. I, in: Franz Kafka, Gesammelte Werke in zwölf Bänden, hrsg. v. Hans-Gerd Koch, a. a. a. O., S. 197 (vgl. auch hier Anmerkung 38).

${ }^{59}$ Dazu ausführlich Bernd Neumann, Franz Kafka. Gesellschaftskrieger, a. a. O., S. $318 \mathrm{ff}$.

${ }^{60}$ Sigmund Freud, Massenpsychologie und Ich-Analyse, in: Gesammelte Werke, hrsg. v. Alexander Mitscherlich, Frankfurt/Main 1982, Bd. IX, S. 83.
} 
Wiener Theorie, angesiedelt in der Trias Sexus, Ruhmverlangen und Assimilation, appliziert auf das Begreifen von ortsgebundener Prager Erfahrung. Die Erkenntnisse Freuds, aus der Konfrontation des Analytikers mit dem „altösterreichischen“ Unterbewusstsein seiner Patienten gewonnen, gleichen in der Tat den Erfahrungen Kafkas mit seinen Prager Vorstädten. Die wurden immer nur widerwillig betreten, etwa, um die Fortschritte der vom Vater gewünschten Fabrik-Gründung zu inspizieren. Im Process-Roman wird K. in eben diesen Vorstädten, auf ihren Dachböden und in ihren Straßen, zu einem buchstäblich Gejagten. Er trifft hier wieder, was er sich selbst im Prozess der assimilatorischen Zivilisation abtrainiert hat, die angstmachende Qualität verdrängter Aggressionen und Gelüste, was ihn zur vorbestimmten ,erotischen“ Beute der nymphenfingrigen Leni macht, der nach meiner Kenntnis einzigen Dame in der Weltliteratur, bei der der Autor die sexuelle Erregung olfaktorisch erfahrbar werden lässt. Die Struktur des von Freud behaupteten psychischen Apparats und die Struktur der besonderen sozialen Energien, die Kafkas Prag durchzogen, gelangen in unserem Denkbild zu gegenseitiger Erhellung. Bei beiden, dem Wiener Freud wie dem Prager Kafka, werden die neu sich bildenden Massen im geschichtlichen Vorfeld der heraufkommenden Totalitarismen zu einer - aufs Neue aktuell gewordenen - Variation jener „Urhorde“, in der einst der sexuelle Anspruch des Hordenführers auf alle Frauen gegolten haben soll. Dies ein Theorem, das sich von Sigmund Freud über Otto Weininger bis hin zu Hans Blüher und dessen Bedeutung für die Konzeption des Schlosses erstreckt - und bereits im Process literarisches, also sinnlich gestaltetes Ereignis wird. Auch dies ein hochemotionaler Komplex, der mit Prag als einem Ort zusammenhing, an dem feindliche Ethnien um die Macht, und darin um die Liebe der Frauen kämpften, in den immer noch ,vergoldeten Jahren“ des sterbenden Doppeladlers, in dessen Rayon zudem die Koordinaten der neuen „Einstein'schen“ Weltsicht sich herauskristallisiert hatten. An sie würde der Prager Autor Franz Kafka sich halten; weshalb er heute noch für jenes weltliterarische Phänomen steht, das sich in ihm verkörperte: Die geglückte Interdependenz zwischen Naturwissenschaft und Literatur, „Erster“ und „Zweiter Kultur“, und darum nur im Lichte einer „Dritten Kultur“ adäquat zu begreifen. Wozu, das wird weiter zu entfalten sein, Kafkas Prager Begegnung mit Albert Einstein entscheidend beigetragen hat. Die wiederum erfolgte zusammen mit Max Brod; erscheint folglich untrennbar mit dessen Tycho Brahe verbunden. 


\subsection{Wer war Kepler in Max Brods Tycho Brahe? Philologische Spurensuche oder Verwirrspiel?}

Max Brod sah in seinem Tycho Brahes Weg zu Gott einen historischen Roman. Das Buch war, wiewohl 1916/1917 in Kriegszeiten erschienen, sein wohl literarischer Erfolg; einer, der ihn gegenüber seinem Verleger Kurt Wolff mit stolzer Genugtuung erfüllt hat. Das Buch hat ein eigenes Kapitel in Michael D. Gordins 2020 erschienenem Einstein in Bohemia erhalten: „The Hidden Kepler“. Dort wird versucht, Kepler zu identifizieren, in einem wahren Kaleidoskop von Namen (Albert Einstein, Franz Werfel, Karl Kraus, auch der andere Franz: nämlich Kafka). Die letzte Sicherheit in der Identifikation ist dabei wohl doch nicht Ereignis geworden. Der Gegenstand könnte zur Satire anregen. Ist dennoch dadurch von Gewicht, dass es bei ihm um „Drittkulturelles“ geht, und wesentlich um die Essenz der Freundschaft zwischen den Prager Dioskuren Brod und Kafka. Das Brod'sche Buch war damals Kafka gewidmet; und ,,antwortete“ darin auf dessen Beschreibung eines Kampfes als seinerseits einer Literarisierung von beider Beziehung. Kafkas genanntes Jugendwerk, deutlich vernachlässigt von der Forschung zugunsten der drei Romane, hatte die Freundschaft zwischen den beiden Freunden als auch eine bittere, von Prags Atmosphäre äußerster kultureller Konkurrenz giftig umnebelte Erzählung über eine Verbindung zu gestalten versucht, deren Kern eigentlich Todfeindschaft sei. ${ }^{61}$ Ein selbst paradoxaler Text mithin, wie gesagt vom Geist radikaler Konkurrenz bestimmt, den man vom stillen Kafka eigentlich nicht erwarten konnte; und dann von Max Brod, so scheint es, mit einem Schopenhauer'schen Verzicht auf Vergeltung beantwortet. Zugrunde lag diesem literarisch ausgetragenem Künstlerstreit unter anderem als Muster: Die in Wien wie Prag allgemein bekannte, ganz besondere Hassbeziehung zwischen Karl Kraus und Max Brod. Die von ersterem als untilgbare ins Leben gerufen worden war mit seinem phänomenal polemischen Satz „Geist auf Brod geschmiert ist Schmalz“. Seitdem hasste man einander glühend als einerseits Kryptokatholik (Kraus) und andererseits tschechisch assimilierter, zionistischer Jude (Brod). In gewisser Weise war das unvermeidbar gewesen. Die Kafka'sche Beschreibung eines Kampfes realisierte lediglich den Sachverhalt: Es konnte immer nur einen geben, Kraus in Wien und Brod in Prag, und weil beider Sphären untrennbar zusammenhingen, musste man sich so hassen, wie

\footnotetext{
${ }^{61}$ Vgl. dazu Bernd Neumann, Franz Kafka. Gesellschaftskrieger. Eine Biografie, München 2008 (Fink), S. 285 ff.
} 
überraschenderweise auch Kafka und Brod in des ersteren Erzählung. Hinzu kam für Brod der unverzeihbare Angriff auf seinen „heiligen“ Prager Freund durch den frivolen Wiener Feind: "Karl Kraus ... attacked and made fun of much evil and many bad authors, but withhout discrimination also did so of great poets like Heine, Werfel, Hofmannsthal, George, or Kafka." "62

Gründe über Gründe also, den Freund zu rächen. Andererseits musste man sich aber auch revanchieren für die Kampfansage der Kafka'schen Kampfbeschreibung, was eine komplizierte literarische Konstruktion erforderlich machte. Die schaute schließlich so aus: Brahe hatte von seinem bewunderten, aber fremden Freund Kepler verlangt, dass dieser, aller Verschiedenheit der beiden zum Trotz (Kepler war bereits Kopernikus-Anhänger, Brahe glaubte noch an Ptolemäus), ihn rächen sollte gegen ein übles Pamphlet, das „Ursus“ (der ein abgefallener Assistent Tychos war und eigentlich Nicolaus Reimer Baer hieß) gegen ihn veröffentlicht hatte, gespickt mit Sottisen und Details aus Brahes Privatsphäre. Kepler folgte dem Wunsch des Meisters, womit er den goldnasigen Dänen noch auf dessen Sterbebett versöhnte - wobei ihm die Bekanntschaft mit dem Golem-Rabbi half, an dessen Beispiel Brods Tycho gelernt hatte, dass Vergebung als die höchste (und eben auch jüdische) Tugend zu betrachten sei. Das fabula docet scheint klar: Der Autor, wie es sich gehörte, identisch mit seiner Hauptfigur, bezeugt also, dass er zu verzeihen gelernt habe, und das als Ausübung der höchsten aller genuin jüdischen Tugenden. Kafka, der stiller, introvertierter als der gesellige Brod, aber dennoch wesentlich bellizistischer war, als assimilierter Jude anverwandelt ,deutscher" Streitlust und Kriegsentschlossenheit (die der Prager in seinen Tagebüchern an den verbündeten Deutschen unübersehbar bewundert hat), Franz Kafka also verhielt sich gegenüber der an ihn gerichtete Widmung des Brod'schen Tycho mit Zurückhaltung. Das geht aus seinen brieflichen Bemerkungen Felice Bauer gegenüber hervor. Kafka teilte der Dauerverlobten nämlich damals mit, daß er und Brod gar nicht mehr so gute, enge Freunde seien; und überhaupt sei Tycho Brahe ein ,sehr persönliches, aber vielleicht auch selbstquälerisches Buch" ${ }^{63}$ Kafka mochte also seine Beschreibung eines Kampfes nicht relativieren, sah gar nicht ein, was daran des Freundes Nachsicht verdiente. Das änderte selbstverständlich nichts an dem Abstand, den auch er Karl Kraus gegenüber einhielt, wobei er damals nicht einmal wissen konnte,

\footnotetext{
${ }^{62}$ Michael D. Gordin, Einstein in Bohemia, a. a. O., S. 166. Den Vorgang hatte Brod selbst, selbstverständlich auf Deutsch, seinerseits in Diesseits und Jenseits veröffentlicht, in Bd. I, S. 331 der 1947 in Winterthur erschienen zweibändigen Ausgabe dieser Schrift.

${ }^{63}$ Brief an Felice, unter dem 20. April 1914, noch vor Kriegsausbruch also abgesandt.
} 
wie die Glorie des Karl Kraus im Berlin der zwanziger Jahre gegenüber dem neuen König dieser Kapitale Bert Brecht sich jämmerlich auflösen würde. ${ }^{64}$ Kafka stand womöglich unter diesem Betracht Brecht näher als Brod, - zumal auch der Stückeschreiber, unter Walter Benjamins Einfluß, die Qualität von Kafkas neuem Schreiben durchaus, wie widerwillig und gespalten auch immer, anerkannt hat. Die wiederum fand ihren Niederschlag auch darin, dass Kepler, verglichen mit dem rastlos lobbyierenden Autor Brod und dessen ungeheurem Bekannten-Netzwerk, eher wie ein stiller, kontemplativer Nachtschreiber wirken konnte. Das drückt die folgende Erkenntnis aus, die bei Gordin zu finden ist (und dessen etwas luftige Spekulationen über Kepler gleich Werfel via Kraus vergessen macht): „Where Tycho, once we meet him, is voluble, impulsive, generous, defensiv, apprehensiv, and in general constantly reacting from moment to moment, Kepler is stasis personified. He is interested in understanding the mechanics of the heavens and persuaded that Copernicus' postulation of the sun as center of the univers is correct. This is bad news for Tycho, who wants his new apprentice to help defend his own cosmology ..."65 Wir aber können uns aus diesem namedropping zwecks Ermittlung des „,verborgenen Kepler“ getrost verabschieden und die Erkenntnis für sicher nehmen, dass Kepler eben der „ist“, dessen Name auch als Widmung auf dem Buche steht: Der Neuerer in allen Belangen des Romans in den Jahren 1915/1916, der Prager Kafka. Als Schöpfer der Blumberg'schen elektromagnetisch vor sich hin springenden Bälle, als Gefährte Max Brods in Prag wie auch in Paris, wo diese elastischen Bälle ebenfalls im Bois de Boulogne hüpften - eben Franz Kafka, dessen Name zwar nicht im, aber auf dem Buche steht. Mit dieser Einsicht in medias res.

\footnotetext{
${ }^{64}$ Dazu im ersten Teil des Buches Bernd Neumann/Gernot Wimmer, Elias Canetti in seiner Zeit, Stuttgart 2020, wo Canetti die Entzauberung des zuvor von ihm zuvor verherrlichten strengen Karl Kraus miterleben musste, betrieben von einem Zigarrenraucher mit Schiebermütze und zynischem Frauenverbrauch, der auch noch einen eigentlich unerschwinglichen Sportwagen fuhr, den der Stückschreiber sich durch gereimte Werbesprüche erdichtet hatte. ${ }^{65}$ Michael D. Gordin, Einstein in Bohemia, a. a. O., S. 153.
} 
Open Access Dieses Kapitel wird unter der Creative Commons Namensnennung 4.0 International Lizenz (http://creativecommons.org/licenses/by/4.0/deed.de) veröffentlicht, welche die Nutzung, Vervielfältigung, Bearbeitung, Verbreitung und Wiedergabe in jeglichem Medium und Format erlaubt, sofern Sie den/die ursprünglichen Autor(en) und die Quelle ordnungsgemäß nennen, einen Link zur Creative Commons Lizenz beifügen und angeben, ob Änderungen vorgenommen wurden.

Die in diesem Kapitel enthaltenen Bilder und sonstiges Drittmaterial unterliegen ebenfalls der genannten Creative Commons Lizenz, sofern sich aus der Abbildungslegende nichts anderes ergibt. Sofern das betreffende Material nicht unter der genannten Creative Commons Lizenz steht und die betreffende Handlung nicht nach gesetzlichen Vorschriften erlaubt ist, ist für die oben aufgeführten Weiterverwendungen des Materials die Einwilligung des jeweiligen Rechteinhabers einzuholen.

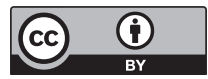

